\title{
Matching ex vivo MRI With Iron Histology: Pearls and Pitfalls
}

\author{
Amaury De Barros ${ }^{1,2 *}$, Germain Arribarat ${ }^{1}$, Jeanne Combis ${ }^{1}$, Patrick Chaynes ${ }^{2}$ and \\ Patrice Péran ${ }^{1}$ \\ ${ }^{1}$ Toulouse Neurolmaging Center, University of Toulouse Paul Sabatier-INSERM, Toulouse, France, ${ }^{2}$ Department of Anatomy, \\ Toulouse Faculty of Medicine, Toulouse, France
}

\section{OPEN ACCESS}

Edited by:

Carol Seger

Colorado State University, United States

Reviewed by:

David Pitt,

Department of Neurology, School of

Medicine, Yale University, United States

Simon Hametner,

Medical University of Vienna, Austria

Kelly M. Gillen,

Department of Radiology, Weill

Cornell Medical College, Cornell

University, United States

*Correspondence:

Amaury De Barros

amaurydebarros@yahoo.fr

Received: 11 April 2019

Accepted: 19 June 2019

Published: 03 July 2019

Citation:

De Barros A, Arribarat G, Combis J, Chaynes $P$ and Péran P (2019)

Matching ex vivo MRI With Iron

Histology: Pearls and Pitfalls.

Front. Neuroanat. 13:68.

doi: 10.3389/fnana.2019.00068
Iron levels in the brain can be estimated using newly developed specific magnetic resonance imaging (MRI) sequences. This technique has several applications, especially in neurodegenerative disorders like Alzheimer's disease or Parkinson's disease. Coupling ex vivo MRI with histology allows neuroscientists to better understand what they see in the images. Iron is one of the most extensively studied elements, both by MRI and using histological or physical techniques. Researchers were initially only able to make visual comparisons between MRI images and different types of iron staining, but the emergence of specific MRI sequences like R2* or quantitative susceptibility mapping meant that quantification became possible, requiring correlations with physical techniques. Today, with advances in MRI and image post-processing, it is possible to look for MRI/histology correlations by matching the two sorts of images. For the result to be acceptable, the choice of methodology is crucial, as there are hidden pitfalls every step of the way. In order to review the advantages and limitations of ex vivo MRI correlation with iron-based histology, we reviewed all the relevant articles dealing with the topic in humans. We provide separate assessments of qualitative and quantitative studies, and after summarizing the significant results, we emphasize all the pitfalls that may be encountered.

Keywords: iron quantification, ex vivo, magnetic resonance imaging, iron histology, QSM

\section{INTRODUCTION}

Iron is an essential cofactor in many physiological processes. In the brain, it contributes to myelination, DNA synthesis, mitochondrial respiration, oxygen transport, and neurotransmitter metabolism (Ward et al., 2014). The iron redox couple $\mathrm{Fe}^{2+} / \mathrm{Fe}^{3+}$ mediates many reactions, the precise redox potential varying according to the nature of the ligand. $\mathrm{Fe}^{2+}$ is essentially present in the labile iron pool in the cytosol, which represents the iron that is directly available for cellular chemistry. $\mathrm{Fe}^{3+}$, which is insoluble, is the major form of iron in the brain, and is mainly found in iron-binding proteins. Hemoproteins (hemoglobin, myoglobin, cytochromes) represent a large proportion of iron in the whole body, but only a minor proportion of iron in the brain parenchyma, except in large vessels. Non-heme iron proteins can be classified according to their function. Transferrin (TF) is the main protein involved in iron uptake by the transferrin-transferrin receptor pathway. TF has two $\mathrm{Fe}^{3+}$ binding sites, but in physiological conditions, only $30 \%$ of TF molecules are loaded (Hare et al., 2013). Transferrin receptor (TfR 1 and 2) are expressed in all the central nervous system and especially in the plasma membrane of the neurons. The non-transferrin bound iron pathway is a minority pathway including several channel proteins and ligand/receptors 
interactions, notably Divalent Metal Transporter 1 (a channel protein) responsible for ferrous iron uptake. Ferroportin (FP) is the only known iron export protein. FP exports $\mathrm{Fe}^{2+}$ from the cytosol, and $\mathrm{Fe}^{2+}$ is further oxidized by hephaestin or ceruloplasmin and their ferroxidase activity. Both are expressed in the brain (Jiang et al., 2017). Hepcidin regulates systemic iron homeostasis by direct interaction with FP. In the cytosol, except in the labile iron pool, iron is ligated by low molecular weight proteins such citrate or ascorbate, iron-sulfur proteins, or ribonucleotides. Ferritin is the major storage protein. It is composed of two subunits, heavy $(\mathrm{H})$ and light $(\mathrm{L})$ chain, that surround a ferrihydrite core capable of storing up to 4,500 iron atoms. In physiological conditions, however, only 2,000-2,500 atoms are usually stored (Bossoni et al., 2017). It should be noted that ferritin may also be iron-free, especially in a pro-inflammatory condition. In the brain, oligodendrocytes (cells producing myelin) are the cells with the most intense immunostaining for ferritin in normal conditions because of their high iron metabolism for axonal myelination. They express both $\mathrm{L}$ and $\mathrm{H}$ subunits, like microglial cells. The neurons essentially express $\mathrm{H}$ subunits. The composition of ferritin in astrocytic cells has yet to be determined (Jiang et al., 2017). Lsubunit is required for the long-term storage of iron, whereas $\mathrm{H}$-subunit has ferroxidase activity. The $\mathrm{H} / \mathrm{L}$ ratio increases with age by a factor 2-3 (Connor et al., 1995).

Magnetite was recently discovered in the brain, and may substitute the ferrihydrite crystal in ferritin in pathological conditions such as Alzheimer's disease (AD) (Bossoni et al., 2017), although this is quite controversial, as some authors claim that the magnetite crystal is too large (Kumar et al., 2016). Magnetite has a magnetic moment 10 times greater than that of ferrihydrite, but seems to be a thousand times less concentrated (Bulk et al., 2018b). Despite its rather misleading name, hemosiderin contains non-heme iron (Harrison and Arosio, 1996). Hemosiderin is regarded as a ferritin degradation product, as immunolabeling studies have found ferritin expressed in siderosomes. Siderosomes are structures that appear to be derived from lysosomes that have degraded ferritin to form hemosiderin in excess iron conditions. In the brain, hemosiderin can be also found during hemorrhages (hematomas, microbleeds, etc.) or in pathological conditions involving excess iron (Harrison and Arosio, 1996; Quintana et al., 2006). Iron is mainly present in the form of ferrihydrite crystals, as it is in ferritin. Hemosiderin is regarded as insoluble, unlike ferritin, and has a high iron/protein ratio. The protein part is less clearly structured than that of ferritin.

Neuromelanin and lipofuscin are two lysosomal products. Neuromelanin is a black, insoluble pigment incorporated into $0.5-3 \mu \mathrm{m}$ intracytoplasmic organelles. These organelles are composed of proteins, lipids and neuromelanin granules of 200$400 \mathrm{~nm}$ with $30-\mathrm{nm}$ substructures. Although the composition and appearance of neuromelanin vary in electron microscopy, depending on the brain region from which it is extracted, the 30-nm substructure is remarkably well-preserved (Zecca et al., 2008; Bush et al., 2009; Engelen et al., 2012). It comprises a eumelanin envelope with a low oxidative potential surrounding a pheomelanin core, with a pheomelanin/eumelanin ratio of
3/1 (Zecca et al., 2008). Neuromelanin is now known to be produced by catecholaminergic neurons in response to excessive cytoplasmic accumulation of unsupported catecholamines in synaptic vesicles. Aside from its role in catecholamines catabolism, neuromelanin is a chelator of heavy metals and environmental toxins (Zucca et al., 2017). In terms of absolute concentration, the most chelated metal by far (by a factor of at least $10^{3}$ relative to the others) is $\mathrm{Fe}^{3+}$. However, the number of chelated metals is extremely varied. It is in the melanincontaining neurons of the susbtantia nigra that we find the most neuromelanin, with a higher iron concentration than elsewhere. These neurons contain very little ferritin, compared with nonmelanin-containing neurons and adjacent glia (Zecca et al., 2004). Neuromelanin has two iron binding sites: a high-affinity site for storing iron in an inactive redox form, which thus participates in its antioxidant action; but also a lower-affinity site that comes into play when the cell is in an iron overload situation. This site is responsible for the easier release of iron and thus contributes to the pro-oxidative properties of neuromelanin. It is now generally accepted that neuromelanin has a dual action (Faucheux et al., 2003; Zucca et al., 2017). There has recently been renewed interest in this substance, partly because of its involvement in the pathophysiology of Parkinson's disease (PD) and related pathologies, and partly because modern imaging techniques allow it to be detected and quantified in vivo, and can thus yield evidence of its variations as a function of time and pathology. This is the case in magnetic resonance imaging (MRI) with fast spin echo techniques involving magnetization transfer (Sasaki et al., 2006, 2008) as well as in positron emission tomography (PET) (Hansen et al., 2016). The iron concentration of neuromelanin has also been experimentally shown to be correlated with T1 and T2 relaxation (Trujillo et al., 2016).

Lipofuscin is another insoluble and ubiquitous pigment (yellow-brown) of the brain. Lipofuscin differs from neuromelanin in its autofluorescence with ultraviolet light (Double et al., 2008). It is also the result of autophagocytosis by the lysosomal system, including autophagocytosis of mitochondria. The final organelle therefore has a size comparable to that of neuromelanin. It is rather perinuclear compared with neuromelanin, which can be found at axonal endings. Lipofuscin increases with age and is a marker of neuronal aging. It is also involved in the chelation of metals such as iron. The presence of free iron contributes to an increase in lipofuscin, probably through lipid peroxidation (Ashraf et al., 2018). Although they would appear to be quite similar, in terms of their formation mechanism and their structure (proteins, lipids, metal ions) lipofuscin and neuromelanin actually seem quite distinct. Moreover, there is no lipofuscin in neurons containing neuromelanin (Terman and Brunk, 2004; Double et al., 2008; Sulzer et al., 2008). There are no scientific data on the potential role of lipofuscin in relaxation in MRI and there are no specific designed sequences for lipofuscin compare to neuromelanin, but we can assume that it does indeed play a role, by virtue of its nature and its proximity to neuromelanin.

In physiological conditions, iron homeostasis is strictly regulated, but in many pathological processes, it is dysregulated, leading to the formation of reactive oxygen species implicated 
in oxidative stress. The main pathologies concerned are extrapyramidal disorders such as PD or multiple system atrophy (MSA), AD, multiple sclerosis (MS), and specific iron metabolism disorders grouped under the heading of neurodegeneration with brain iron accumulation (Dusek et al., 2012). Aging is the main factor favoring accumulation of iron in the brain. Iron increases during the first decades, reaching maximum values during the fifth or sixth decade with only subtle changes after (HALLGREN and SOURANDER, 1958).

\section{IRON DETECTION USING HISTOLOGICAL OR PHYSICAL TECHNIQUES}

Ever since the pioneering work carried out around the turn of the Twentieth-century by Zaleski (1887) and Spatz (1922) (Koeppen, 1995), brain iron quantification and characterization has been an important topic of research. Histological methods such as Perls' or Turnbull staining are the best known techniques. Perls' stain reacts mainly with $\mathrm{Fe}^{3+}$ ions, giving an intense Prussian (or Berlin) blue. However, it also reacts with $\mathrm{Fe}^{2+}$, resulting in a white precipitate known as Everitt's salt that slowly oxidizes to give Prussian blue (Meguro et al., 2007). Perls' staining only makes it possible to highlight non-heme iron. The iron present in the heme has strong covalent bonds and only treatment with strong alkalis (ammonium sulfide) can dissociate it (Meguro et al., 2007). By contrast, the iron bound to iron metabolism molecules (ferritin, hemosiderin, transferrin, iron-sulfur proteins, etc.) can easily be ionized from a $\mathrm{pH}$ of around 4.5. This explains why treatment with hydrochloric acid is necessary for Perls' staining. Like iron heme, iron present in neuromelanin and probably lipofuscin is historically described as invisible iron, owing to its strong affinities. As a result, it cannot be revealed by Perls' staining. The second best known histochemical method is the Turnbull technique, which consists in applying potassium ferricyanide instead of potassium ferrocyanide, again in association with hydrochloric acid. This treatment makes it possible to reveal $\mathrm{Fe}^{2+}$ ions. Other methods involve using the iron-sulfur reaction to demonstrate both $\mathrm{Fe}^{2+}$ and $\mathrm{Fe}^{3+}$. Treatment with ammonium sulfide reveals $\mathrm{Fe}^{2+}$ (Quincke method), and pretreatment with ammonium sulfide and secondary treatment with Turnbull stain reveal $\mathrm{Fe}^{3+}$ in addition to $\mathrm{Fe}^{2+}$ (Tirmann-Schmelzer method). The TirmannSchmelzer method is an iron-sulfur method. Theoretically, the iron-sulfur technique has three drawbacks. First, not all $\mathrm{Fe}^{3+}$ is converted to $\mathrm{Fe}^{2+}$, thus limiting the sensitivity of the technique. Second, ammonium sulfide treatment also extracts heme iron in theory but only if the treatment is at particular low $\mathrm{pH}$ and/or for a very long period (several days) compare to classical protocols (90 min). Third, sulfur-based methods are not specific for iron and causes the precipitation of other metal ions, such as copper or zinc into copper sulfide or zinc sulfide. However, these precipitates do not further react with potassium ferricyanide (Turnbull reaction). So, while the simple iron-sulfur techniques react with zinc and copper, the actual Tirmann-Schmelzer method does not suffer from this lack of specificity, because the specificity for iron comes from the reaction of $\mathrm{Fe}^{2+}$ ions



with potassium ferricyanide (Meguro et al., 2007). The TirmannSchmelzer method has recently been used to study iron in rigorous studies with good results (Wiggermann et al., 2017; Hametner et al., 2018).

To enhance the visualization (augmented sensitivity) of Perls' or Turnbull staining, diaminobenzidine (DAB) can be added, turning Perls' or Turnbull's blue to a brown palette (cf. Figure 1). Another colorimetric assay method involves reacting ferrozine with $\mathrm{Fe}^{2+}$ to give a magenta red precipitate that can then be assayed spectrophotometrically. Pretreatment with hydroxylamine hydrochloride converts $\mathrm{Fe}^{3+}$ to $\mathrm{Fe}^{2+}$.

Although the iron itself cannot be revealed by immunohistochemistry, the same is not true of the proteins containing this iron. The advantage of this technique is that it identifies antigens (generally proteins) of interest at the cellular or extracellular level using specific antibodies. These antibodies may be of either polyclonal or monoclonal origin. Polyclonal antibodies recognize various antigens of the protein of interest. There is therefore a risk of cross-reactions. Monoclonal antibodies recognize a single antigen. They have greater specificity, but diminished sensitivity.

The main proteins that can be targeted are transferrin, FP, iron-responsive proteins, and ferritin. There are both polyclonal and monoclonal antibodies for ferritin. However, each antibody is specific for either the $\mathrm{L}$ chain, the $\mathrm{H}$ chain, or mitochondrial ferritin.

In conclusion, there are no perfect staining methods for accurately calculating the iron concentration of a given tissue. They are therefore considered to be semiquantitative methods at best. Their strength lies in their qualitative character to highlight areas rich in iron with an excellent spatial resolution, whether macroscopically or microscopically.

Physical techniques like inductively coupled plasma mass spectrometry (ICP-MS) or atomic absorption spectroscopy (AAS) are used to quantify iron concentrations with precision, but they cannot map the iron in the brain. Recent techniques such as laser ablation inductively coupled plasma mass spectrometry (LA-ICP-MS) or X-ray fluorescence (XRF) can robustly quantify and precisely map iron in brain structures (Matusch et al., 2011; Zheng et al., 2013). 


\section{MR SIGNAL AND IRON-BINDING PROTEINS}

Ferritin, hemosiderin and deoxyhemoglobin are considered to be the main forms of iron present in sufficient quantities to affect MR contrast in the human brain (Schenck, 2003; Haacke et al., 2005). To a lesser extent, neuromelanin can also affect the MRI signal in particular locations. Lipofuscin's signal impact needs more investigation. Proteins associated with small numbers of iron atoms like transferrin are often regarded as negligible. Consider the case of ferritin. It is not so much its total composition that is important as its core, most often a ferrihydrite crystal which, like all metallic nanoparticles, has magnetic properties capable of affecting susceptibility. Ferrihydrite is an antiferromagnetic structure with, by definition, antiparallel magnetic ordering with moment compensation. The magnetization vector in a crystal is not isotropic, as the direction of spin relative to the crystal lattice depends on magnetocrystalline anisotropy. This anisotropy stems from the presence of easy magnetization directions within the crystal. However, the nanometric size of the core (about $6 \mathrm{~nm}$ ) gives it different properties. Below a certain size, particle magnetization relaxes above the energy barriers of anisotropy. If the volume of the particle is small enough, the energy barriers separating the minimum energy directions (easy directions) may correspond to thermal energy at room or body temperature. The magnetic moment can fluctuate from one easy direction to another. This thermally activated phenomenon constitutes superparamagnetic relaxation (Daou, 2007; Bennett et al., 2008).

It should also be noted that the particle's magnetic moment depends on the number of iron atoms and surface/volume ratio in ferritin. The maximum capacity is 4,500 atoms, but the actual capacity often oscillates around 2,000 atoms. Finally, the crystal is not always in the form of ferrihydrite, as it can also be in the form of magnetite/maghemite, which has a magnetic moment 10 times greater than the crystal of ferrihydrite.

Magnetic susceptibility, denoted as chi $(\chi)$, is defined as the ratio of the magnetic moment $(\mathrm{M})$ to the magnitude of the external magnetic field (B). This variable is dimensionless.

$$
\chi=M / B
$$

Its molar magnetic susceptibility $(\chi \mathrm{m})$ is equal to its magnetic susceptibility $(\chi)$ multiplied by the molar mass of the test compound.

Diamagnetic substances have a molar susceptibility of $<1$ ppm. This is the case of most human tissue, which is very weakly diamagnetic. By contrast, paramagnetic and superparamagnetic substances have a molar susceptibility $>1 \mathrm{ppm}$, and some substances, such as ferritin, have a very high molar susceptibility (5,000 ppm).

In other words, highly (super- or ferro-) paramagnetic substances significantly increase the magnetic field at the local level. This increase in the magnetic field results in changes in the frequency of spin precession, and thus in a poor location of the MR signal (image distortion). These local inhomogeneities of the magnetic field also have the effect of shortening the $\mathrm{T} 2{ }^{*}$ or $\mathrm{T} 2$ times, by accelerating the spin phase shifts in between.

This is called the susceptibility artifact. Magnetic susceptibility, phase shift and shortening of $\mathrm{T} 2 *$ are the main approaches to the quantification of iron in MRI.

\section{IRON ESTIMATION BY MRI}

Advances in MRI mean that we are now able to estimate the presence of iron in the brain. Several techniques have emerged to quantify iron content. Some are based on transverse relaxation time (T2, T2* and T2') or its inverse (R2, R2* and $\mathrm{R} 2^{\prime}$ ), in the relation $\mathrm{R} 2^{*}=\mathrm{R} 2+\mathrm{R} 2$ '. Iron, especially when it is stored in ferritin or hemosiderin, induces local magnetic field inhomogeneities and increases $\mathrm{R} 2$ and $\mathrm{R} 2 *$ relaxation rates. Transverse relaxation rates $(\mathrm{R} 2$ or $\mathrm{R} 2 *$ ) can be measured by multi-echo acquisition (spin echo for $\mathrm{R} 2$ and gradient echo for R2*), then voxelwise fitting to an exponential model (Péran et al., 2007).

The second type of sequences used to estimate iron are based on paramagnetic effects from iron-containing proteins that increase bulk tissue magnetic susceptibility. Magnetic susceptibility information is contained in phase images. Susceptibility weighted imaging (SWI), which combines phase images with magnitude images to enhance contrast among structures with different local susceptibilities, was the first to be used. Quantitative susceptibility mapping (QSM) was developed more recently to directly measure susceptibility and be independent of imaging parameters such as echo time or field strength (de Rochefort, 2010; Deistung et al., 2016). QSM is based on phase images and uses complex posttreatment algorithms to remove background field components after phase unwrapping. Several algorithms and methods can be used, such as Laplacian-based methods, calculation of susceptibility using multiple orientation sampling (COSMOS), morphology-enabled dipole inversion (MEDI), and sophisticated harmonic artifact reduction on phase data (SHARP) method (Wang and Liu, 2015; Deistung et al., 2016). QSM allows investigators to distinguish between calcium and iron, unlike R2*. There are other sequences, such as field-dependent R2 increase (FDRI), that have high sensitivity and specificity for iron, but they are seldom used in clinical practice, owing to technical constraints.

Iron-based sequences are used in research or clinical routine in healthy participants (Péran et al., 2009) to estimate the amount of iron in brain structures, especially the basal ganglia, or to compare patients with diseases such as PD with healthy controls (Péran et al., 2010) or establish differential diagnoses for disorders such as MSA (Barbagallo et al., 2016; Péran et al., 2018).

\section{IRON-MRI CORRELATION}

Researchers trying to correlate iron-based sequences with an estimation of iron concentration in brain structures draw on data from a seminal work published in 1958 by HALLGREN 


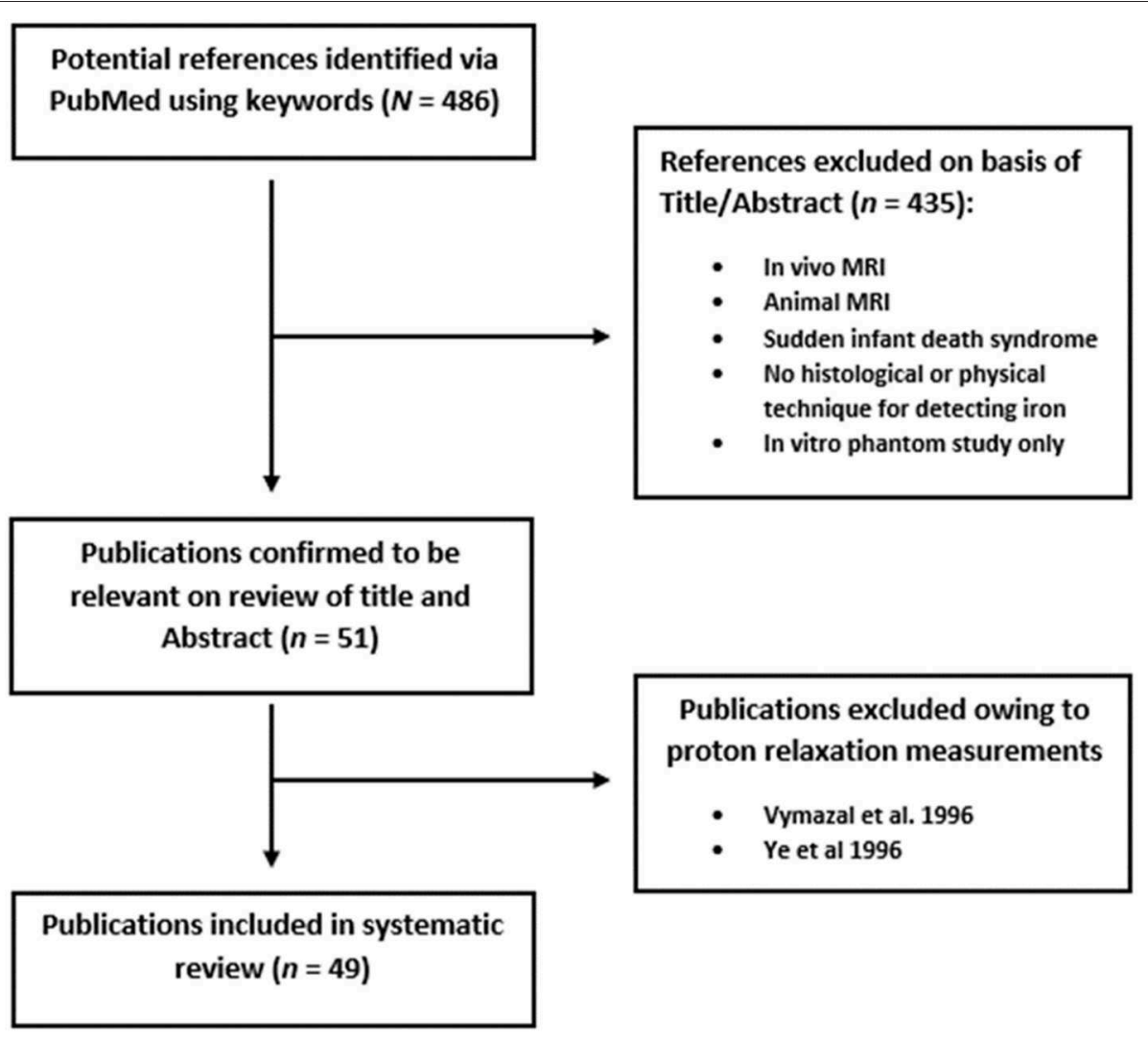

FIGURE 2 | Flowchart depicting systematic review process.

and SOURANDER (1958) and verified in recent research (Krebs et al., 2014), using regression line equations for many parts of the brain, where $y=$ concentration of non-heme iron per $100 \mathrm{~g}$ fresh weight and $\mathrm{x}=$ age of participant. For all types of iron-based sequences [FDRI Bartzokis et al., 2007,R2*Péran et al., 2009, or QSM Bilgic et al., 2012], correlations are calculated with Hallgren and Sourander's equations.

Despite these rigorous data, the desire to correlate estimations of iron with MRI using a reference quantitative technique has led scientists to undertake ex vivo MRI, which allows for further investigations after scanning. Ex vivo MRI has many advantages compared with in vivo MRI, including longer acquisition time for better spatial resolution, air-tissue interface elimination, no importance of the specific absorption rate (SAR), no patient artifacts, and the possibility of using a dedicated receiver coil for small anatomical specimens. Ex vivo MRI has gained in popularity in the scientific community, not only for anatomical atlases and forensic medicine, but also for molecular and trace element mapping.

The aim of the present literature review was to systematically describe and evaluate published studies that used ex vivo MRI combined with histological or physical techniques to detect iron in the brain. We focused on quantitative studies that attempted to find statistical correlations between the two kinds of techniques, but also included articles exploring iron in relation to a specific pathology or just for anatomical description purposes. After summarizing their results, we discuss all the pitfalls that can be encountered along the way.

\section{METHODS}

The initial search was undertaken on PubMed using the following terms: "brain" AND "magnetic resonance imaging" OR "MRI" OR "MR" OR “QSM" OR "R2*” AND "Ex-vivo" OR "postmortem" OR "postmortem” AND “iron." The review was carried out in accordance with PRISMA guidelines for systematic reviews (Moher et al., 2009). Our search criteria yielded 486 results. We limited the articles we reviewed to human studies and papers written in English. Relevant papers were identified by manual review of titles and abstracts. Articles with no evidence of iron staining or detection by a physical technique on the MR scanned specimen were excluded. A total of 51 articles were fully analyzed, and two were excluded owing to the use of proton relaxation measurements rather than MRI (Vymazal et al., 1996; Ye et al., 1996). We distinguished quantitative analyses from qualitative (visual assessment) ones in the 49 final articles and listed all the different pathologies concerned (Figure 2). Relevant data were listed. In the case of the quantitative studies, we identified the linear regression parameters, along with their linear equation parameters. Finally, key methodological points were noted, in order to highlight limitations that may be encountered when matching ex vivo MRI with histology. 


\section{RESULTS}

Table 1 sums up all the recent literature on post mortem MRI and histological or physical techniques for measuring iron. Papers were classified as quantitative ( 25 articles) when correlations were sought between MR data and histological or physical techniques, and qualitative (24 articles) when images were simply visually assessed. Where articles described two reference techniques (often a histological one and a physical one), the quantitative assessment was only applied to the physical technique. In some papers, quantitative measures were carried out in order to compare data between the pathological group and controls, so they were not classified as quantitative.

MR scanners varied widely in main field strength (1.421.1 tesla), with the largest, supported by micro-MRI, being particularly useful for small specimens. Many MRI techniques were used to highlight iron, but two recent techniques (R2* and QSM) seemed particularly valuable. A total of 17 articles published after 2009 featured R2* techniques (Yao et al., 2009; Fukunaga et al., 2010; Langkammer et al., 2010; Bagnato et al., 2011, 2018; Antharam et al., 2012; Kwan et al., 2012; Langkammer, 2012; Walsh et al., 2013; Stüber et al., 2014; Dusek et al., 2015; Meadowcroft et al., 2015; Wisnieff et al., 2015; Birkl et al., 2017; Bulk et al., 2018b; Hametner et al., 2018), while 10 published after 2012 used QSM techniques (Langkammer et al., 2012; Zheng et al., 2013; Stüber et al., 2014; Tan et al., 2014; Sun et al., 2015; Wisnieff et al., 2015; Wiggermann et al., 2017; Hametner et al., 2018; Lee et al., 2018; Kaunzner et al., 2019). However, many more techniques (T1 mapping, T2, T2 mapping, R2, R2', SWI, phase, etc.) were linked more or less successfully with iron. Some authors worked on the relation between transverse relaxation time (R2* and R2') and specimen temperature, developing an iron quantification method based on a temperature coefficient (Birkl et al., 2015, 2017). This seems a very interesting approach to investigating the relationship between MR signal, iron, myelin and temperature, and is a good example of what can be done with ex vivo material compared with in vivo. This technique now needs to be reproduced by other teams to confirm its effectiveness.

The types of anatomical specimens used differed greatly. Some studies used excisional material from surgery, such as gangliogliomas or cavernomas, while others compared pathological samples from a brain bank with healthy controls, or fresh specimens from required autopsies or anatomy laboratories. Anatomical specimens also differed in size, with some teams using the whole brain, many others only a thick coronal slice (often from brain banks) and the remainder using a small anatomical part, such as the substantia nigra, putamen, subthalamic nucleus, or medium temporal gyrus.

Regarding pathologies, AD (11 articles), MS (9 articles), PD (3 articles), progressive supranuclear paralysis (PSP) (3 articles), MSA (2 articles) and amyotrophic lateral sclerosis (2 articles) were the most frequently studied conditions.

Regarding the physical techniques used for iron quantification, ICP-MS was the one most often used (6 articles), with LA-ICP-MS performed in one study (Wisnieff et al., 2015). The second most frequent technique (4 articles) allowing for mapping was XRF. An interesting technique based on the substance's magnetic characteristics, known as superconducting quantum interference device magnetometry (SQUID), was used to further characterize the paramagnetic properties of the particles, allowing, for example, ferrihydrite crystal to be differentiated from magnetite/maghemite (Birkl et al., 2015; Bulk et al., 2018b).

Given that physical techniques require expensive materials, physicians, expert technicians and sound knowledge of physics, histology remains of interest because of its low cost, simplicity and opportunities for mapping. Perls' staining was the most frequently used technique (19 articles for Perls and 8 articles for Perls $+\mathrm{DAB})$, followed by Turnbull $+\mathrm{DAB}+$ ammonium sulfide (5 articles).

Table 2 sums up all the different combinations of MRI and histological or physical techniques.

Although correlations between in vivo MRI (especially R2* and QSM) and Hallgren's equations yielded remarkably reproducible results across the different teams (Ghassaban et al., 2018), many centers tried to confirm these by correlating MRI and histological/physical data. We selected 25 articles because one of the goals of the studies they described was to quantify iron using a histological/physical technique and correlate it with MRI sequences. All these studies are summarized in Table 3. Relevant MRI sequence acquisition parameters are provided. When available, Pearson $(r)$ or Spearman $(\rho)$ correlation coefficients or coefficients of determination $\left(r^{2}\right)$ are given in Column 6 with their associated $p$-value. Linear equation parameters are set out in Column 7. As only relevant or overall results are given, they do not reflect all the correlations found in the studies.

Quantitative correlations have been attempted with many physical techniques, but advances in digitization and image postprocessing software have recently led to a renewal of interest in Perls' or Turnbull staining. Table 4 lists all the articles describing MRI + Perls' or Turnbull staining. Staining techniques and slice thickness are provided, along with the digitization step and a brief description of the correlation methodology. Visual always refers to qualitative assessment.

\section{DISCUSSION}

\section{Qualitative Histological Studies and MRI}

Since the advent of MRI, constant efforts have been made to improve understanding of what the images show. Iron is a perfect candidate in this respect, as its paramagnetic property means that it is observable by MRI. Furthermore, iron may play a role in many neurological disorders. With the aim of better understanding what we can see in in vivo MRI and being certain that it is iron, it is obviously useful to look for correlations between MRI sequences and iron histology.

In the case of AD, Dhenain et al. (2002) and House et al. (2008) failed to correlate iron in senile plaques with $\mathrm{T} 2 *$ hypointensities or R1 and R2, in contrast to van Rooden et al. (2009), who concluded that amyloid plaques and cerebral amyloid angiopathy (CAA) are collocated with iron. Meadowcroft et al. (2015) confirmed the role of iron in the transverse relaxation rate 
TABLE 1 | List of all references featuring iron descriptions by both MRI and histological/physical technique.

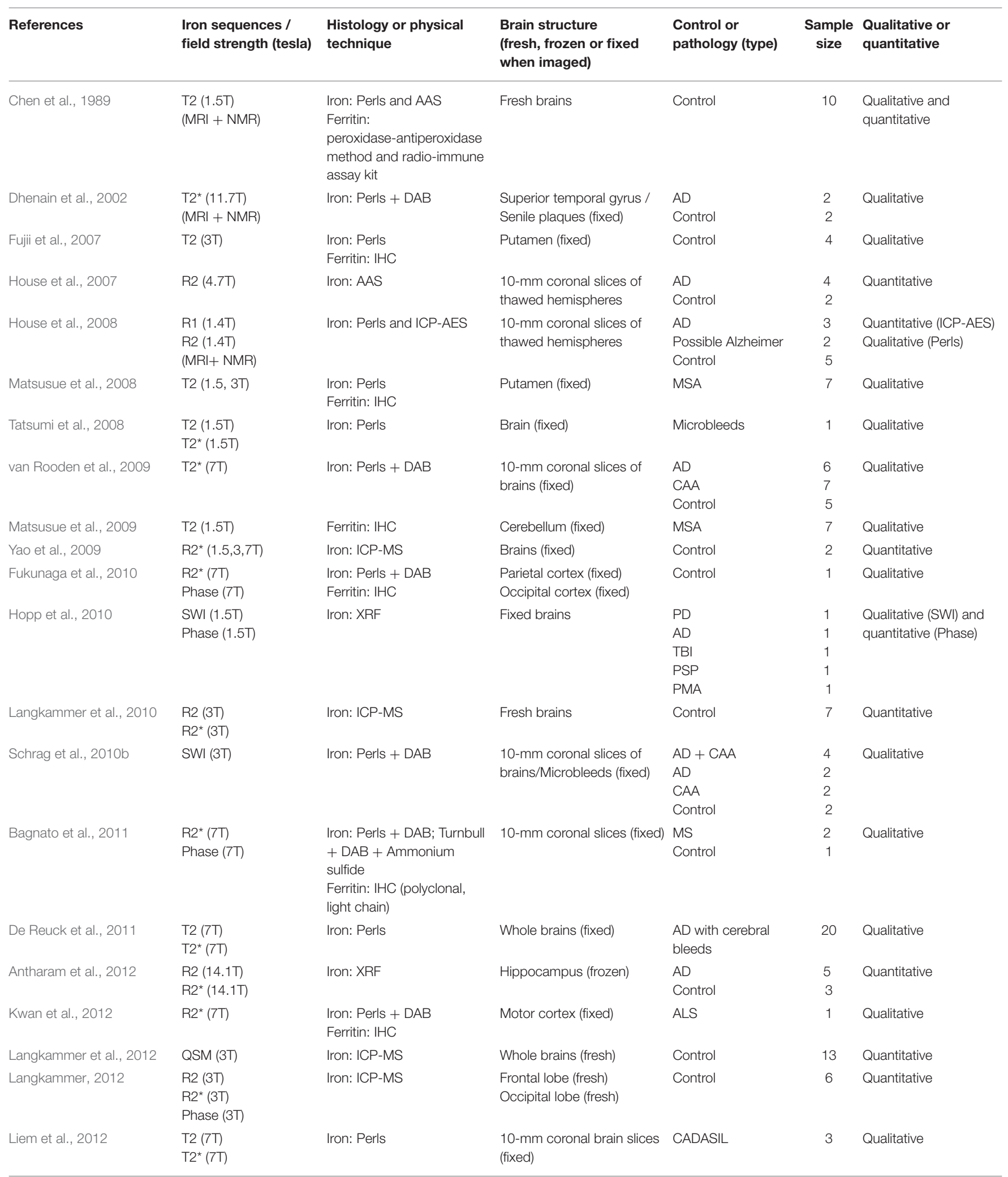


TABLE 1 | Continued

\begin{tabular}{|c|c|c|c|c|c|c|}
\hline References & $\begin{array}{l}\text { Iron sequences / } \\
\text { field strength (tesla) }\end{array}$ & $\begin{array}{l}\text { Histology or physical } \\
\text { technique }\end{array}$ & $\begin{array}{l}\text { Brain structure } \\
\text { (fresh, frozen or fixed } \\
\text { when imaged) }\end{array}$ & $\begin{array}{l}\text { Control or } \\
\text { pathology (type) }\end{array}$ & $\begin{array}{l}\text { Sample } \\
\text { size }\end{array}$ & $\begin{array}{l}\text { Qualitative or } \\
\text { quantitative }\end{array}$ \\
\hline Zheng et al., 2012 & $\begin{array}{l}\text { T2* }(3 T) \\
\text { Flair }(3 T) \\
\text { SWI }(3 T)\end{array}$ & Iron: XRF & $\begin{array}{l}\text { 10-mm coronal brain slices } \\
\text { (fixed) }\end{array}$ & Stroke & 3 & Qualitative \\
\hline Foroutan et al., 2013 & $\begin{array}{l}\text { T2 }(21.1 \mathrm{~T}) \\
\mathrm{T}^{*}(21.1 \mathrm{~T})\end{array}$ & $\begin{array}{l}\text { Iron: Perls } \\
\text { Ferritin: IHC (light chain) }\end{array}$ & $\begin{array}{l}\text { Basal ganglia and midbrain } \\
\text { (fixed) }\end{array}$ & $\begin{array}{l}\text { PSP } \\
\text { Control }\end{array}$ & $\begin{array}{l}6 \\
3\end{array}$ & Qualitative \\
\hline Walsh et al., 2013 & $\begin{array}{l}\mathrm{R} 2(4.7 \mathrm{~T}) \\
\mathrm{R} 2^{*}(4.7 \mathrm{~T}) \\
\text { Phase }(4.7 \mathrm{~T})\end{array}$ & Iron: Perls & $\begin{array}{l}\text { Basal ganglia and MS } \\
\text { lesions (fresh) }\end{array}$ & MS & 3 & Quantitative \\
\hline Stüber et al., 2014 & $\begin{array}{l}\text { R1 }(7 T) \\
R 2^{*}(7 T) \\
\text { QSM }(7 T)\end{array}$ & Iron: Perls and PIXE & $\begin{array}{l}\text { Occipital lobe (fixed) } \\
\text { Paracentral cortex (fixed) } \\
\text { STN (fixed) }\end{array}$ & Control & 3 & $\begin{array}{l}\text { Quantitative (PIXE) } \\
\text { Qualitative (Perls) }\end{array}$ \\
\hline Tan et al., 2014 & QSM (3T) & Iron: ICP-MS & Cavernomas (fixed) & Cavernomas & 4 & Quantitative \\
\hline Birkl et al., 2015 & $\begin{array}{l}\text { R2' (3T) } \\
\text { TCR2' (3T) }\end{array}$ & $\begin{array}{l}\text { Iron: SQUID magnetometry } \\
\text { and ICP-MS }\end{array}$ & Whole brain (fresh) & Control & 5 & Quantitative \\
\hline Sun et al., 2015 & QSM (4.7T) & Iron: Perls & Whole brain (fresh) & Control & 2 & Quantitative \\
\hline Wisnieff et al., 2015 & $\begin{array}{l}\mathrm{R}^{*}(3 T) \\
\text { QSM (3T) }\end{array}$ & Iron: Perls and LA-ICP-MS & MS lesions (fixed) & MS & 1 & $\begin{array}{l}\text { Quantitative } \\
\text { (LA-CP-MS) } \\
\text { Qualitative (Perls) }\end{array}$ \\
\hline Dusek et al., 2016 & $\begin{array}{l}\mathrm{T} 2^{*}(7 \mathrm{~T}) \\
\mathrm{R}^{*}(7 \mathrm{~T})\end{array}$ & $\begin{array}{l}\text { Iron: Turnbull + DAB + } \\
\text { Ammonium sulfide and AAS } \\
\text { Ferritin: IHC }\end{array}$ & Basal ganglia (fixed) & $\begin{array}{l}\text { Wilson } \\
\text { Control }\end{array}$ & 96 & $\begin{array}{l}\text { Quantitative } \\
\text { Qualitative (Ferritin) }\end{array}$ \\
\hline Dal-Bianco et al., 2017 & $\begin{array}{l}\text { T2 }(7 T) \\
\text { Phase }(7 T) \\
\text { SWI }(7 T)\end{array}$ & Iron: Turnbull + DAB & MS lesions (fixed) & MS & 4 & Qualitative \\
\hline De Reuck et al., 2017 & $\mathrm{~T}^{*}(7 \mathrm{~T})$ & Iron: Perls & Coronal brain slices (fixed) & $\begin{array}{l}\text { ALS } \\
\text { FTLD } \\
\text { Control }\end{array}$ & $\begin{array}{l}12 \\
38 \\
28\end{array}$ & Qualitative \\
\hline Massey et al., 2017 & T2 (9.4T) & Iron: Perls & Substantia nigra (fixed) & $\begin{array}{l}\text { Control } \\
\text { PD } \\
\text { PSP }\end{array}$ & $\begin{array}{c}10 \\
5 \\
8\end{array}$ & Qualitative \\
\hline Matsuda et al., 2017 & $\begin{array}{l}\text { T1 }(7 \mathrm{~T}) \\
\text { T2 }(7 \mathrm{~T}) \\
\mathrm{T}^{*}(7 \mathrm{~T})\end{array}$ & Iron: Perls & Ganglioglioma (fixed) & Ganglioglioma & 1 & Qualitative \\
\hline Wiggermann et al., 2017 & QSM (3T) & $\begin{array}{l}\text { Iron: Turnbull + ammonium } \\
\text { sulfide + DAB }\end{array}$ & MS lesions (fixed) & MS & 5 & Quantitative \\
\hline Bagnato et al., 2018 & $\mathrm{R}^{*}(7 \mathrm{~T})$ & $\begin{array}{l}\text { Iron: Turnbull + DAB + } \\
\text { Ammonium sulfide }\end{array}$ & $\begin{array}{l}\text { 10-mm coronal brain slices } \\
\text { (fixed) }\end{array}$ & MS & 7 & Quantitative \\
\hline Bulk et al., 2018b & $\mathrm{R}^{*}(7 \mathrm{~T})$ & Iron: EPR and SQUID & $\begin{array}{l}\text { Medium temporal gyrus } \\
\text { (fixed) }\end{array}$ & $\begin{array}{l}\mathrm{AD} \\
\text { Control }\end{array}$ & $\begin{array}{l}1 \\
1\end{array}$ & Quantitative \\
\hline
\end{tabular}




\begin{tabular}{|c|c|c|c|c|c|c|}
\hline Bulk et al., $2018 \mathrm{a}$ & $\mathrm{T}^{*}(7 \mathrm{~T})$ & Iron: Perls + DAB & Medium frontal gyrus (fixed) & $\begin{array}{l}A D \\
\text { Control }\end{array}$ & $\begin{array}{l}21 \\
13\end{array}$ & Quantitative \\
\hline Hametner et al., 2018 & $\begin{array}{l}\mathrm{R}^{*}(7 \mathrm{~T}) \\
\mathrm{QSM}(7 \mathrm{~T})\end{array}$ & $\begin{array}{l}\text { Iron: Turnbull + ammonium } \\
\text { sulfide + DAB and ferrozine } \\
\text { assay }\end{array}$ & Whole brain (fresh) & Control & 6 & Quantitative \\
\hline Kaunzner et al., 2019 & QSM (3T) & Iron: Perls + DAB & MS lesions & MS & 7 & Qualitative \\
\hline Lee et al., 2018 & $\begin{array}{l}\text { T1map } \\
\text { T2map } \\
\text { T2*map } \\
\mathrm{T}^{*} / \mathrm{T} 2 \\
\mathrm{~T} 2^{*} / \mathrm{T}^{2} \\
\mathrm{SWI} \\
\mathrm{QSM}\end{array}$ & Iron: Perls & Substantia nigra (fixed) & Control & 5 & Quantitative \\
\hline
\end{tabular}

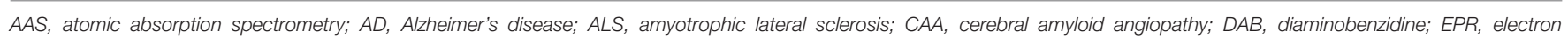

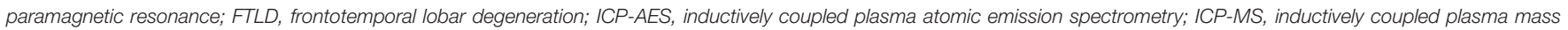

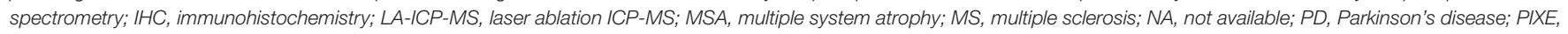

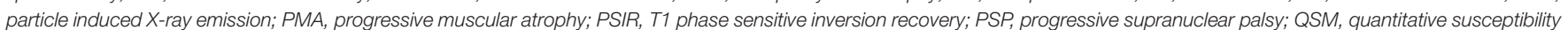

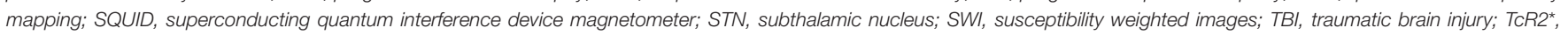
temperature coefficient $R 2^{\star}$; $X R F, X$-ray fluorescence.

in $A \beta$ plaques, but when they compared deferoxamine ironchelated tissue with unchelated tissue using Perls + DAB, they demonstrated that iron is not the sole determinant of the relaxation rate, and the fibrillar nature of $A \beta$ plaques also has a major role in transverse relaxation. Bulk et al. (2018a) demonstrated a good spatial correlation between iron revealed by Perls $+\mathrm{DAB}$ and $\mathrm{T}^{*} *$ in the whole cortex of patients with $\mathrm{AD}$, and not only in $\mathrm{A} \beta$ plaques as previously described. They compared early-onset $\mathrm{AD}$ with late-onset, and showed that iron-rich microglial cell recruitment is responsible for a higher iron concentration in the early-onset stage than in controls or the late-onset stage. They confirmed that iron is not the sole determinant of $\mathrm{T} 2{ }^{*}$-weighted contrast, and that myelin also plays a decisive role, with a good spatial correlation between iron and myelin as already described elsewhere (Fukunaga et al., 2010; Wallace et al., 2016). In another article, Bulk et al. (2018b) described significantly higher values of R2*, a high concentration of ferrihydrite, and a higher magnetic moment of magnetite/maghemite particles in the middle temporal gyrus of patients with $\mathrm{AD}$.

Schrag et al. (2010b) found a correlation between SWI and hemosiderin in brain microbleeds in patients with CAA and AD that was subsequently confirmed by De Reuck et al. (2011) and van Veluw et al. (2016), with correlations between T2/T2*and Perls. Interestingly, some acute hemorrhages were not stained, unlike old hemorrhages, confirming the absence of heme-iron staining by Perls and the gradual breakdown of blood products into hemosiderin.

For PD, Blazejewska et al. (2013) described an overlapping of Perls' staining with $\mathrm{T}^{*}$ hypointensity after image coregistration in the substantia nigra of patients with PD and controls. Regions with high neuromelanin did not correlate with Perls' staining and $\mathrm{T} 2 *$ hypointensities, confirming that iron stored in neuromelanin cannot be stained by Perls, probably owing to its strong affinity with neuromelanin proteins (Zucca et al., 2017). The authors found that nigrosome- 1 had a low iron concentration, which was confirmed by Massey et al. (2017), with no differences between patients with PD and controls, compared with patients with PSP, in whom nigrosome-1 appears hyperintense in Perls' staining. In patients with PSP, relative to controls, Foroutan et al. (2013) also described an increase in iron in the substantia nigra and globus pallidus highlighted both by T2* and by Perls. In MSA, (Matsusue et al., 2008, 2009) revealed ferritin deposits in the dentate nucleus that were associated with $\mathrm{T} 2 *$ weighted image hypointensity. In the putamen, hypointensities on $\mathrm{T} 2 *$ were also correlated with ferritin and extensive iron staining.

From a purely anatomical point of view, besides the low concentration of iron in nigrosome-1 evoked earlier, more intense staining was reported in the anteromedial part of the substantia nigra pars reticulata where pallido-striato-nigral fibers begin (Blazejewska et al., 2013; Lee et al., 2016, 2018; Massey et al., 2017). This was confirmed by another study using LAICP-MS (Matusch et al., 2011). In the subthalamic nucleus, ironrich regions were found to be located in the anteromedial part of the nucleus, with iron levels gradually decreasing toward the posterolateral part. This was assessed both by MRI-Perls analysis (Massey et al., 2012) and by other techniques (Dormont et al., 2004; de Hollander et al., 2014).

For Wilson's disease, Dusek et al. (2016) found that iron and copper levels were higher in the putamen of patients than controls, but only iron concentration was correlated with R2*.

In MS, Bagnato et al. (2011) compared R2* and phase sequences at $7 \mathrm{~T}$ with Perls $+\mathrm{DAB}$ staining and several antibodies for immunohistochemistry (ferritin, oligodendrocytes, microglia...). They found that both oligodendrocytes and microglia were iron-rich in the normal-appearing white 
TABLE 2 | Summary of all combinations of MRI sequences with histological or physical technique.

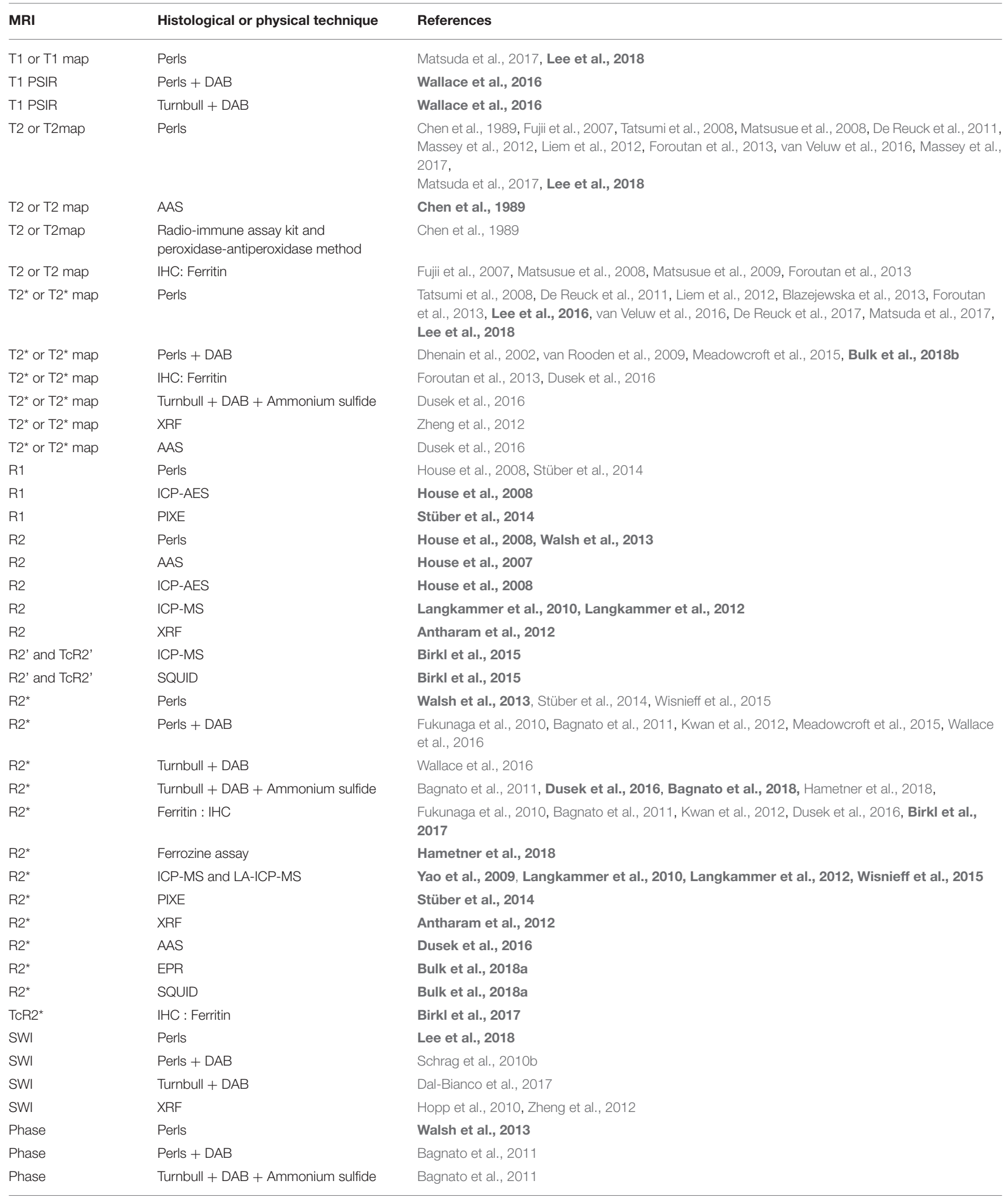


TABLE 2 | Continued

\begin{tabular}{|c|c|c|}
\hline MRI & Histological or physical technique & References \\
\hline Phase & IHC: Ferritin & Bagnato et al., 2011 \\
\hline Phase & ICP-MS & Langkammer et al., 2012 \\
\hline Phase & $\mathrm{XRF}$ & Hopp et al., 2010 \\
\hline QSM & Perls & Stüber et al., 2014, Sun et al., 2015, Wisnieff et al., 2015, Lee et al., 2018 \\
\hline QSM & Perls + DAB & Kaunzner et al., 2019 \\
\hline QSM & Turnbull + DAB + Ammonium sulfide & Wiggermann et al., 2017, Hametner et al., 2018 \\
\hline QSM & ICP-MS and LA-ICP-MS & Langkammer, 2012, Tan et al., 2014, Wisnieff et al., 2015 \\
\hline QSM & XRF & Zheng et al., 2013 \\
\hline QSM & Ferrozine assay & Hametner et al., 2018 \\
\hline
\end{tabular}

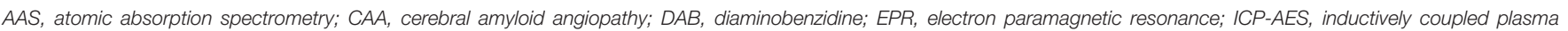

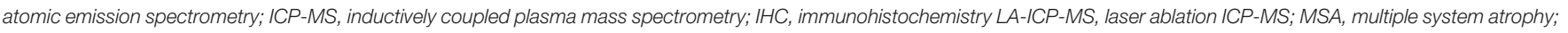

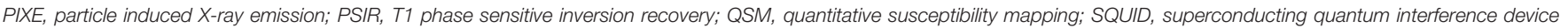
magnetometer; SWI, susceptibility weighted images; TCR2*, temperature coefficient R2*; XRF, X-ray fluorescence. Quantitative correlations are indicated in bold.

matter of patients with MS, compared with oligodendrocytes only in controls. Ferritin- and iron-enriched activated microglia/macrophages were also found surrounding the core of the lesion, but not in the core itself, except in the perivascular space where extracellular hemosiderin can be found. When Walsh et al. (2013) explored 30 MS lesions from three different patients, they found heterogeneous patterns of Perls' staining and R2* and phase signal in these lesions. Wisnieff et al. (2015) concluded that iron is responsible for positive susceptibility values (QSM) in demyelinated lesions, but in normal-appearing white matter, the myelin contribution prevents the analysis of iron by QSM. The ferritin count also differs between the core and the edge of the lesions, with more ferritin in the edge, as revealed by Birkl et al. (2017). According to Wiggermann et al. (2017), few MS lesions contain iron diffusively, and QSM should not be interpreted in terms of the lesions' bulk magnetic susceptibility, but relative to abnormalities in the surrounding normal-appearing white matter. Bagnato et al. (2018) confirmed that both iron and myelin contribute to the $\mathrm{R} 2 *$ signal, but in different ways, depending on which brain structure is evaluated. They stated that iron is a stronger contributor to R2* changes than myelin. We are of the view that it is fiber orientation more than myelin quantity that plays a role in $\mathrm{R} 2 *$ variations. For an extended review of MS and iron, see Stüber et al. (2016), in which a diagram based on lesion chronology tries to explain the various patterns found both in iron histology and in iron-MRI sequences. These authors also point out that the basal ganglia appear richer in iron in patients with MS than in controls.

We can see that MRI/histology visual comparisons can be a mine of information and provide a set of data that are helpful for clinicians and neuroscientists and which cannot be obtained with other techniques. However, visual comparisons are often more subjective than strict quantitative correlations.

\section{Quantitative Histological Studies With Physical Techniques and MRI}

Physical techniques are regarded as the benchmark for detecting trace elements such as iron. House et al. (2008), House et al.
(2007) were the first to find a correlation between relaxation parameters (R1, R2) and iron concentration, as determined by AAS or by inductively coupled plasma atomic emission spectrometry (ICP-AES) $[r=0.72$ (R1) and 0.70-0.797 (R2)]. Results for R2 are remarkably consistent across studies, regardless of which technique is used: $r=0.67$ with ICP-MS (Langkammer et al., 2010); $r=0.69$ with XRF (Antharam et al., 2012). Linear correlation equation parameters are of the same order of magnitude. For R2 ${ }^{*} r$ ranges from 0.37 to 0.93 , with a significant $p$-value, in line with in vivo studies (Ghassaban et al., 2018). The degree of correlation depends on which anatomical structure is studied and the number and exhaustiveness of the sampling points. For example, when Langkammer (2012) studied the frontal and occipital gray/white matter interface, conducting correlation analyses of both gray and white matter with iron determined by ICP-MS, they found significant correlations both overall $(r=0.37, p<0.01)$ and for gray matter only $(r=0.42$, $p<0.05)$, but not for white matter only. They postulated that R2* is affected both by paramagnetic iron and by diamagnetic myelin. By contrast, when they pooled the basal ganglia with white matter, the Pearson correlation coefficient rose to $r=$ $0.90(p<0.001)$, owing to the richness in iron and relative poorness in myelin of the basal ganglia (Langkammer et al., 2010). To overcome the myelin contribution, Stüber et al. (2014) separated the myelin fraction and iron concentration determined by particle-induced X-ray emission in the paracentral lobule and occipital lobe. A univariate analysis with iron yielded a correlation of $r=0.56$, but a multivariate analysis with myelin fraction and iron concentration yielded a correlation of $r=$ 0.93. This clearly demonstrated that iron and myelin are the two main contributors to $\mathrm{R} 2 *$ in white matter. The authors claimed that fiber orientation contributes to the $\mathrm{R} 2 *$ relaxation rate compared with the $\mathrm{R} 1$ rate, which is affected by myelin quantity but not orientation. When Yao et al. (2009) plotted R2* values as a function of field strength, they demonstrated that R2* augments with field strength, and R2* rate of change with field strength is a function of iron concentration $(r=0.93)$. They concluded that R2* depends linearly on iron concentration, at all field strengths studied (1.5-3-7 T). Bulk et al. (2018b) 
TABLE 3 | List of all quantitative studies with corresponding correlation parameters.

\begin{tabular}{|c|c|c|c|c|c|c|}
\hline References & $\begin{array}{l}\text { Iron quantification } \\
\text { method/field strength } \\
\text { (tesla) }\end{array}$ & 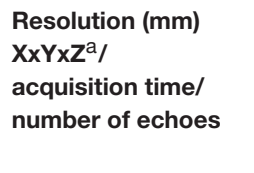 & $\begin{array}{l}\text { Fixation period } \\
\text { before MRI }\end{array}$ & $\begin{array}{l}\text { Histological } \\
\text { or } \\
\text { physical technique }\end{array}$ & $\begin{array}{l}\text { Linear regression arameters } \\
r=\text { Pearson coefficient } \\
\text { correlation } \\
r^{2}=\text { coefficient of } \\
\text { determination }\end{array}$ & $\begin{array}{l}\text { Linear correlation } \\
\text { equation } \\
\mathrm{A} \times[\mathrm{Fe}]+\mathrm{B}\end{array}$ \\
\hline Chen et al., 1989 & T2 (1.5T) & $\begin{array}{l}5 \\
\text { NA } \\
\text { NA }\end{array}$ & 0 & Iron: AAS & $p=0.1$ & No correlation \\
\hline House et al., 2007 & $\mathrm{R} 2(4.7 \mathrm{~T})$ & $\begin{array}{l}0.6 \times 0.3 \times 4 \\
\text { NA } \\
7\end{array}$ & 0 & Iron: AAS & $r=0.797, p<0.0001$ & $\begin{array}{l}A=0.098 \\
B=16.2\end{array}$ \\
\hline House et al., 2008 & $\begin{array}{l}\text { R1 }(1.4 \mathrm{~T}) \\
\text { R2 }(1.4 \mathrm{~T})\end{array}$ & $\begin{array}{l}\text { NA } \\
\text { NA } \\
\text { NA }\end{array}$ & 0 & Iron: ICP-AES & $\begin{array}{l}\text { R1: } r=0.72, p<0.0001 \\
\text { R2: } r=0.70, p<0.0001\end{array}$ & $\begin{array}{l}R 1: A=0.0034, B=0.9 \\
R 2: A=0.031, B=10.0\end{array}$ \\
\hline Yao et al., 2009b & $\operatorname{R}^{*}(1.5,3,7 \mathrm{~T})$ & $\begin{array}{l}0.7 \times 0.7 \times 0.7 \\
\text { 3h } 00 \mathrm{~min} 00 \mathrm{~s} \\
5\end{array}$ & NA & Iron: ICP-MS & $r=0.93, p<0.05^{b}$ & $\begin{array}{l}A=0.0099^{b} \\
B=1.71\end{array}$ \\
\hline Langkammer et al., 2010 & $\begin{array}{l}\mathrm{R} 2(3 \mathrm{~T}) \\
\mathrm{R} 2^{*}(3 \mathrm{~T})\end{array}$ & $\begin{array}{l}1 \times 1 \times 4 \\
\text { Oh } 06 \min 27 \mathrm{~s} \\
32 \\
1 \times 1 \times 4 \\
\text { Oh } 07 \mathrm{~min} 35 \mathrm{~s} \\
12\end{array}$ & 0 & Iron: ICP-MS & $\begin{array}{l}\text { R2: } r=0.67, p<0.001 \\
\mathrm{R}^{*}: r=0.90, p<0.001\end{array}$ & $\begin{array}{l}R 2: A=0.04, B=8.5 \\
R 2^{*}: A=0.27, B=14.3\end{array}$ \\
\hline Hopp et al., 2010 & Phase (1.5T) & $\begin{array}{l}0.5 \times 0.5 \times 2 \\
\text { NA } \\
\text { NA }\end{array}$ & NA & Iron: XRF & Phase: $r^{2}=0.81, p<0.0001$ & $\begin{array}{l}\text { Phase: } A=0.0025, B= \\
-0.13\end{array}$ \\
\hline Antharam et al., 2012 & $\begin{array}{l}\text { R2 (14.1T) } \\
\text { R2* }(14.1 T)\end{array}$ & $\begin{array}{l}0.062 \times 0.062 \times 0.08 \\
1 \mathrm{~h} 40 \mathrm{~min} 00 \mathrm{~s} \\
15 \\
0.062 \times 0.062 \times 0.08 \\
1 \mathrm{~h} 30 \mathrm{~min} 00 \mathrm{~s} \\
18\end{array}$ & 0 & Iron: XRF & $\begin{array}{l}\text { R2: } r^{2}=0.48 \\
\text { R2* }: r^{2}=0.43\end{array}$ & $\begin{array}{l}\text { NA } \\
\text { NA }\end{array}$ \\
\hline Langkammer, 2012 & QSM (3T) & $\begin{array}{l}0.5 \times 0.5 \times 2 \\
0 \mathrm{~h} 18 \mathrm{~min} 00 \mathrm{~s} \\
\text { NA }\end{array}$ & 0 & Iron: ICP-MS & $r=0.87, p<0.001$ & $A=0.00097, B=-0.037$ \\
\hline Langkammer et al., 2012 & $\begin{array}{l}\mathrm{R}^{*}(3 \mathrm{~T}) \\
\text { Phase (3T) }\end{array}$ & $\begin{array}{l}0.5 \times 0.5 \times 2 \\
0 \mathrm{~h} 17 \min 27 \mathrm{~s} \\
2\end{array}$ & 0 & Iron: ICP-MS & $\begin{array}{l}\mathrm{R} 2^{*}: r=0.37 p<0.01 \\
\text { Phase: } r=-0.14, p=0.33\end{array}$ & $\begin{array}{l}R 2^{*}: A=0.12, B=22.38 \\
\text { Phase: NA }\end{array}$ \\
\hline Walsh et al., 2013 & $\begin{array}{l}\text { T2 }(4.7 \mathrm{~T}) \\
\text { R2 }(4.7 \mathrm{~T}) \\
\text { R2* }(4.7 \mathrm{~T}) \\
\text { Phase }(4.7 \mathrm{~T})\end{array}$ & $\begin{array}{l}0.25 \times 0.25 \times 1-2 \\
\text { Oh } 06 \min 48 \mathrm{~s} \\
\text { NA } \\
0.8-1 \times 1 \times 4-5 \\
\text { Oh } 15 \min 36 \mathrm{~s} \\
18-24 \\
0.8-1 \times 1 \times 2\end{array}$ & 0 & Iron: Perls & $\begin{array}{l}\text { T2: } r^{2}=0.511-0.650 p<0.001 \\
\text { R2: } r^{2}=0.489-0.615 p<0.001 \\
\text { R2 }: r^{2}=0.628-0.685 p< \\
\text { 0.001 } \\
\text { Phase: } r^{2}=0.441-0.596 p \\
<0.001\end{array}$ & $\begin{array}{l}\text { NA } \\
\text { NA } \\
\text { NA } \\
\text { NA }\end{array}$ \\
\hline
\end{tabular}


TABLE 3 | Continued

\begin{tabular}{|c|c|c|c|c|c|c|}
\hline References & $\begin{array}{l}\text { Iron quantification } \\
\text { method/field strength } \\
\text { (tesla) }\end{array}$ & $\begin{array}{l}\text { Resolution (mm) } \\
X x Y \times Z^{a} / \\
\text { acquisition time/ } \\
\text { number of echoes }\end{array}$ & $\begin{array}{l}\text { Fixation period } \\
\text { before MRI }\end{array}$ & $\begin{array}{l}\text { Histological } \\
\text { or } \\
\text { physical technique }\end{array}$ & $\begin{array}{l}\text { Linear regression arameters } \\
r=\text { Pearson coefficient } \\
\text { correlation } \\
r^{2}=\text { coefficient of } \\
\text { determination }\end{array}$ & $\begin{array}{l}\text { Linear correlation } \\
\text { equation } \\
\mathrm{A} \times[\mathrm{Fe}]+\mathrm{B}\end{array}$ \\
\hline & & $\begin{array}{l}\text { Oh } 08 \min 54 \mathrm{~s} \\
10 \\
0.5 \times 0.5 \times 2 \\
\text { Oh } 06 \min 36 \mathrm{~s} \\
\text { NA }\end{array}$ & & & & \\
\hline Zheng et al., 2013 & QSM (3T) & $\begin{array}{l}0.5 \times 0.5 \times 0.7 \\
\text { NA } \\
11\end{array}$ & 6 hours & Iron: XRF & $r=0.74-0.87 p=N A$ & $\begin{array}{l}A=0.79-0.80 \\
B=-3.66-+10.81\end{array}$ \\
\hline Stüber et al., $2014^{C}$ & $\begin{array}{l}\mathrm{R} 1(7 \mathrm{~T}) \\
\mathrm{R} 2^{*}(7 \mathrm{~T}) \\
\mathrm{QSM}(7 \mathrm{~T})\end{array}$ & $\begin{array}{l}0.2 \times 0.2 \times 0.2 \\
\text { NA } \\
2 \\
0.2 \times 0.2 \times 0.2 \\
\text { NA } \\
8 \\
0.2 \times 0.2 \times 0.2 \\
\text { NA } \\
8\end{array}$ & 30 days & Iron: PIXE & $\begin{array}{l}\mathrm{R}^{\mathrm{C}}: r=0.871 p<0.001 \\
\mathrm{R}^{* \mathrm{C}}: r=0.933 p<0.001 \\
\mathrm{QSM}^{\mathrm{C}}: r=0.812 p<0.001\end{array}$ & $\begin{array}{l}A=0.249^{d}, B=1.129 \\
A=0.0526^{d}, B=45.87 \\
A=0.000143^{d}, \\
B=-0.0251\end{array}$ \\
\hline Tan et al., 2014 & QSM (3T) & $\begin{array}{l}\mathrm{NA} \times \mathrm{NA} \times 0.4 \\
\text { Oh } 02 \mathrm{~min} 29 \mathrm{~s} \\
\text { NA }\end{array}$ & NA & Iron: ICP-MS & $r=0.86 p<0.01$ & NA \\
\hline Birkl et al., 2015 & $\begin{array}{l}\text { R2' (3T) } \\
\text { TcR2' (3T) }\end{array}$ & $\begin{array}{l}1 \times 1 \times 2 \\
\text { Oh } 20 \min 0 \mathrm{~s} \\
6\left(\mathrm{~T}^{*}\right)\end{array}$ & 0 & Iron: ICP-MS & $\begin{array}{l}\text { R2': } r^{2}=0.94 p<0.001 \\
\text { TcR2': } r^{2}=0.98 p<0.001\end{array}$ & $\begin{array}{l}A=0.11, B=8.85 \\
A=-0.004, B=0.03\end{array}$ \\
\hline Sun et al., 2015 & QSM (4.7T) & $\begin{array}{l}0.8-1 \times 1 \times 2 \\
0 \mathrm{~h} 08 \min 54 \mathrm{~s} \\
10\end{array}$ & 0 & Iron: Perls & $r^{2}=0.62-0.86 p<0.001$ & NA \\
\hline Wisnieff et al., 2015 & $\begin{array}{l}R 2^{*}(3 T) \\
\text { QSM (3T) }\end{array}$ & $\begin{array}{l}0.7 \times 0.7 \times 0.7 \\
\text { NA } \\
12\end{array}$ & NA & Iron: LA-ICP-MS & NA & NA \\
\hline Dusek et al., 2016 & $\mathrm{R}^{*}(7 \mathrm{~T})$ & $\begin{array}{l}0.5 \times 0.5 \times 2 \\
0 \text { h } 35 \mathrm{~min} 00 \mathrm{~s} \\
8\end{array}$ & NA & $\begin{array}{l}\text { Iron:Turnbull }+ \text { DAB }+ \\
\text { ammonium sulfide and } \\
\text { AAS }\end{array}$ & $\begin{array}{l}\text { Turnbull: } r^{2}=0.57-0.64 p \\
=0.01-0.02 \text { AAS: } r^{2}=0.70, p \\
<0.001\end{array}$ & NA \\
\hline Lee et al., 2016 & $\mathrm{~T}^{\star}(7 \mathrm{~T})$ & $\begin{array}{l}0.136 \times 0.136 \times 0.5 \\
\text { Oh } 25 \mathrm{~min} 0 \mathrm{~s} \\
10\end{array}$ & 3-5 months & Iron: Perls & $r=-0.45--0.63 p<0.001$ & $\begin{array}{l}A=-8.05--12.64^{e} \\
B=10.83-11.95 e^{e}\end{array}$ \\
\hline Wallace et al., 2016 & PSIR & $\begin{array}{l}0.3 \times 0.3 \times 0.6 \\
6 \mathrm{~h} 35 \mathrm{~min} 0 \mathrm{~s} \\
\text { NA }\end{array}$ & Several months & $\begin{array}{l}\text { Iron: Perls + DAB and } \\
\text { Turnbull + DAB }\end{array}$ & $r=0.18 p=\mathrm{NA}$ & NA \\
\hline Birkl et al., 2017 & $\mathrm{R}^{*}(3 \mathrm{~T}) \mathrm{TcR} 2^{*}(3 \mathrm{~T})$ & $\begin{array}{l}0.5 \times 0.5 \times 2 \\
0 \mathrm{~h} 06 \min 28 \mathrm{~s}\end{array}$ & NA & Ferritin: IHC (light chain) & $\begin{array}{l}\mathrm{R}^{*}: r=0.14 p=0.36 \\
\text { TcR2 }: r=-0.66 p<0.001\end{array}$ & $\begin{array}{l}A=0.06, B=37.51 \\
A=-0.0032, B=-0.7756\end{array}$ \\
\hline
\end{tabular}


TABLE 3 | Continued

\begin{tabular}{|c|c|c|c|c|c|c|}
\hline References & $\begin{array}{l}\text { Iron quantification } \\
\text { method/field strength } \\
\text { (tesla) }\end{array}$ & 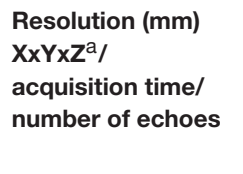 & $\begin{array}{l}\text { Fixation period } \\
\text { before MRI }\end{array}$ & $\begin{array}{l}\text { Histological } \\
\text { or } \\
\text { physical technique }\end{array}$ & $\begin{array}{l}\text { Linear regression arameters } \\
r=\text { Pearson coefficient } \\
\text { correlation } \\
r^{2}=\text { coefficient of } \\
\text { determination }\end{array}$ & $\begin{array}{l}\text { Linear correlation } \\
\text { equation } \\
\mathrm{A} \times[\mathrm{Fe}]+\mathrm{B}\end{array}$ \\
\hline & & 6 & & & & \\
\hline Wiggermann et al., 2017 & QSM (3T) & $\begin{array}{l}0.4 \times 0.4 \times 0.8 \\
0 \mathrm{~h} 14 \mathrm{~min} 20 \mathrm{~s} \\
\text { NA }\end{array}$ & NA & $\begin{array}{l}\text { Iron: Turnbull }+ \\
\text { ammonium sulfide }+ \\
\text { DAB }\end{array}$ & $r^{2}=0.02 p=0.35$ & NA \\
\hline Bagnato et al., 2018 & $\mathrm{R}^{*}(7 \mathrm{~T})$ & $\begin{array}{l}0.7 \times 0.7 \times 0.7 \\
2 \mathrm{~h} 16 \mathrm{~min} 52 \mathrm{~s} \\
5\end{array}$ & Several years & $\begin{array}{l}\text { Iron: Turnbull + DAB+ } \\
\text { ammonium sulfide }\end{array}$ & $r=0.42-0.86 p<0.01$ & NA \\
\hline Bulk et al., 2018a & $\mathrm{R}^{*}(7 \mathrm{~T})$ & $\begin{array}{l}\times 0.1 \times 0.1 \\
3 \mathrm{~h} 30 \mathrm{~min} 0 \mathrm{~s} \\
4\end{array}$ & 4 weeks -3 years & Iron: EPR and SQUID & $\rho=0.12^{f} p>0.05$ & NA \\
\hline Bulk et al., 2018b & $\mathrm{T}^{*}(7 \mathrm{~T})$ & $\begin{array}{l}\text { / } \\
3 \mathrm{~h} 30 \mathrm{~min} 0 \mathrm{~s}\end{array}$ & NA & Iron: Perls + DAB & $r=0.6 p=\mathrm{NA}$ & NA \\
\hline Lee et al., 2018 & $\begin{array}{l}\text { T1map (7T) } \\
\text { T2map (7T) } \\
\text { T2*map (7T) } \\
\text { T2*/T2 (7T) } \\
\mathrm{T} 2^{*} / \mathrm{T} 2^{2} \\
(7 \mathrm{~T}) \\
\text { SWI (7T) } \\
\text { QSM (7T) }\end{array}$ & $\begin{array}{l}0.136 \times 0.136 \times 0.5 \\
\text { NA } \\
10-15\end{array}$ & NA & Iron: Perls & $\begin{array}{l}\text { T1map: } r=-0.37--0.11 p< \\
\text { 0.001 } \\
\text { T2map: } r=-0.61--0.35 p< \\
0.001 \\
\text { T2*map: } r=-0.65--0.11 p< \\
0.001 \\
\text { T2*/T2: } r=-0.64--0.15 p< \\
0.001 \\
\text { T2*/T22 }: r=-0.43--0.26 p< \\
0.001 \\
\text { SWI: } r=-0.65--0.18 p< \\
\text { 0.005 } \\
\text { QSM: } r=0.29-0.44 p<0.001\end{array}$ & NA \\
\hline Hametner et al., 2018 & $\begin{array}{l}\mathrm{R} 2^{*}(7 \mathrm{~T}) \\
\mathrm{QSM}(7 \mathrm{~T})\end{array}$ & $\begin{array}{l}0.43 \times 0.43 \times 0.65 \\
0 \mathrm{~h} 13 \mathrm{~min} 10 \mathrm{~s} \\
4\end{array}$ & 0 & $\begin{array}{l}\text { Iron: Turnbull }+ \\
\text { ammonium sulfide + } \\
\text { DAB } \\
\text { Ferrozine assay }\end{array}$ & $\begin{array}{l}\text { Turnbull: R2*: } r=0.619 p< \\
0.0001 \\
\text { Turnbull: QSM: } r=0.445 p< \\
0.0001 \\
\text { Ferrozine: R2*: } r=0.738 p=\text { NA } \\
\text { Ferrozine: QSM: } r=0.755 p \\
=\text { NA }\end{array}$ & $\begin{array}{l}A=0.51236, B=15.2827 \\
A=0.0006723 B= \\
-0.04618 \\
A=1.10, B=-7.13 \\
A=0.00196 B=-0.10\end{array}$ \\
\hline
\end{tabular}

AAS, atomic absorption spectrometry; CAA, cerebral amyloid angiopathy; DAB, diaminobenzidine; EPR, electron paramagnetic resonance; ICP-AES, inductively coupled plasma atomic emission spectrometry; ICP-MS, inductively coupled plasma mass spectrometry; IHC, immunohistochemistry; LA-ICP-MS, laser ablation ICP-MS; MSA, multiple system atrophy; NA, not available; PIXE, particle induced X-ray emission; PSIR, T1 phase sensitive inversion recovery; QSM, quantitative susceptibility mapping; SQUID, superconducting quantum interference device magnetometer; SWI, susceptibility weighted images; TcR2*, temperature coefficient R2*; XRF, X-ray fluorescence.

a: $X \times Y$, in-plane resolution; $Z$, thick section

b: iron correlated with $R 2^{*} /$ tesla rather than $R^{*}$

c: linear equation parameters determined with myelin inference

d: correlation with iron expressed in $\mathrm{kg} / \mathrm{mg}$ dry weight determined by PIXE

e: linear equation parameters determined with neuromelanin inference

f: Spearman correlation coefficient. 
TABLE 4 | Technical description of Perls' and Turnbull staining with brief description of correlation methodology with MRI.

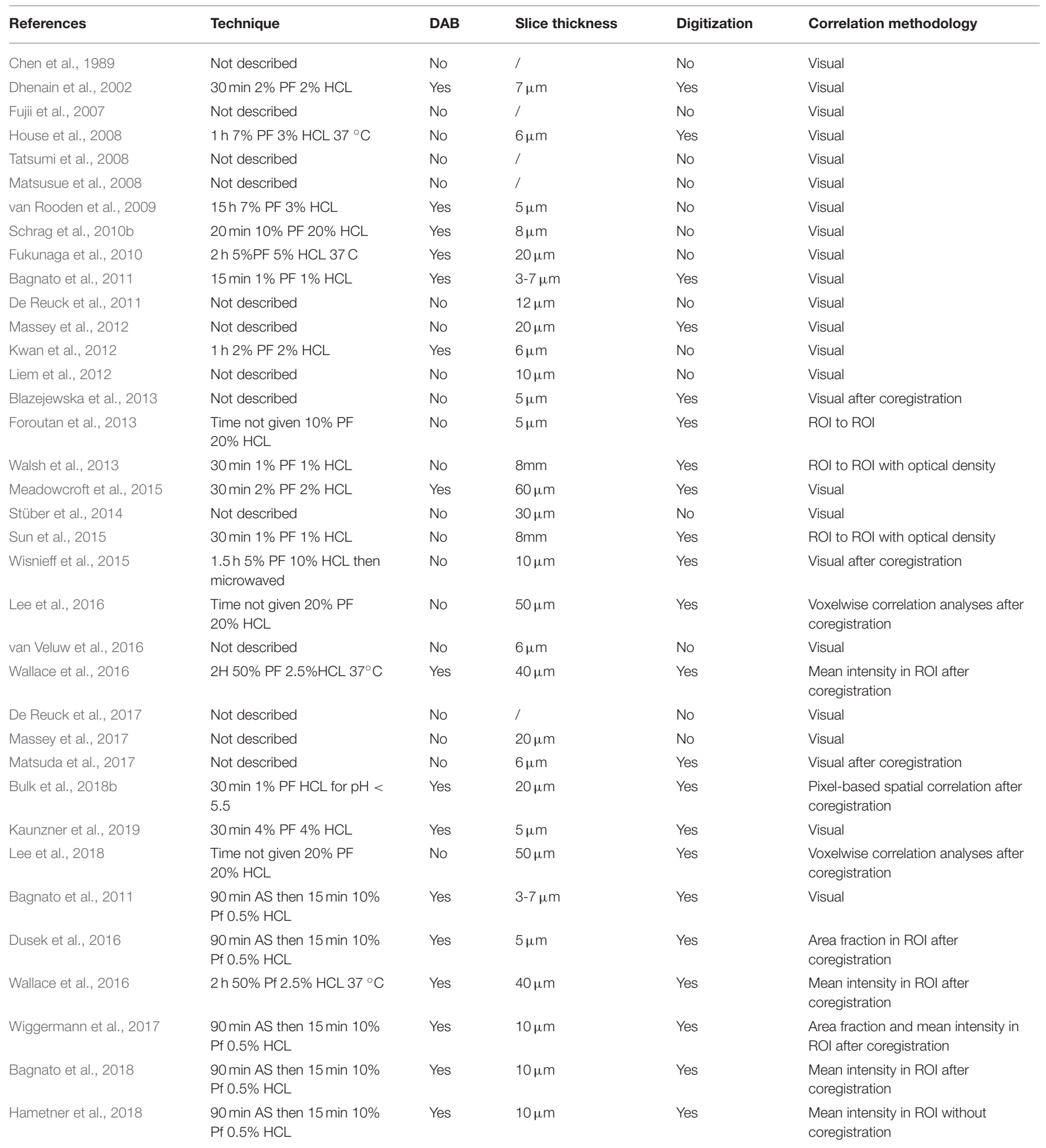

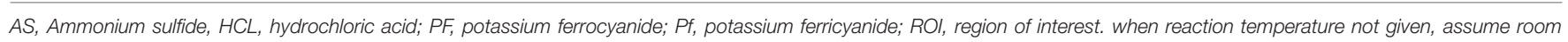
temperature. Solid line separates Perls' procedures (above) from Turnbull procedures (below).

failed to find a correlation between iron $\left(\mathrm{Fe}^{3+}\right)$ measured by electron paramagnetic resonance (EPR) or ferrihydrite and magnetite/maghemite measured by SQUID and $2^{*}$ in the temporal cortex. This can be explained by the choice of anatomical specimen and by various technical considerations identified by the authors. 
Hopp et al. (2010) found a strong correlation between phase images and XRF $(r=0.90, p<0.0001)$, whereas Langkammer (2012) failed to do so. The main difference between these two studies was the exhaustiveness of the sampling. Hopp et al. used several parts of the brain, including the basal ganglia, whereas Langkammer et al. only used the frontal and occipital cortices with their adjacent white matter (owing to their research question). Using QSM, Langkammer et al. (2012) sampled basal ganglia and lobar white matter offering a wide range of iron levels, as measured by ICP-MS. The resulting correlation appeared strong ( $r=0.87, p<0.001)$. Zheng et al. (2013) used pixel-by-pixel correlation allowed by XRF mapping to confirm this correlation ( $r=0.74$ for right side and $r=0.87$ for left side). However, they only considered basal ganglia (putamen, caudate nucleus, and globus pallidus) from one individual in the analysis. Other studies have reported correlations ranging from $r=0.76$ to $r=0.86$ (Stüber et al., 2014; Tan et al., 2014; Hametner et al., 2018), in accordance with in vivo studies (Ghassaban et al., 2018). The QSM method these authors used included homogeneityenabled incremental dipole inversion (Langkammer et al., 2012; Hametner et al., 2018), threshold k-space division (TKD) (Zheng et al., 2013), morphology enabled dipole inversion (MEDI) (Tan et al., 2014; Wisnieff et al., 2015), and calculation of susceptibility through multiple orientation sampling (COSMOS) (Wisnieff et al., 2015).

Ex vivo quantitative studies performed in different brain subregions and with different physical techniques confirm in vivo correlations made with Hallgren and Sourander's equations, especially for R2* and QSM. In the basal ganglia and cortex, iron accounts for most of the recorded signal, compared with white matter in which myelin quantity and anisotropy form part of the signal.

Mapping is difficult to perform with physical techniques, and has so far only been undertaken by a few research teams. Technical progress in image digitization now makes it possible to perform MR signal correlations directly with colorimetry.

\section{Quantitative Histological Studies With Staining and MRI}

We have seen how a robust protocol is essential for quantitative studies, but it is truer still for studies with staining. Colorimetry can be described as a semiquantitative tool, as it can only provide an indirect estimation based on optical density or pixel intensity, not a direct concentration of an element (iron). However, the same could be said of all available MRI techniques that only quantify a signal (relaxation rate, susceptibility), rather than directly measuring iron concentrations. Despite these considerations, some authors, using a good image coregistration methodology, have successfully demonstrated the utility of histology/MRI correlation that does not solely rely on simple visual assessment.

Walsh et al. (2013) compared R2, R2*, T2 and phase imaging with Perls' staining on 8-mm slices through the whole brain. Each slice was photographed before and after staining. For each set of slice photographs, grayscale conversion was performed, adjusting the window and level of the stained photographs to match those of the unstained photographs using two reference points: background and a region of unstained white matter. The differences in intensity between the stained and unstained photographs were then normalized after division by the difference in intensity between the background and the unstained white-matter reference region for each slice. An image of relative optical density was thus produced, where a higher value corresponded to greater iron staining. Several regions of interest (ROIs) in the basal ganglia were used for analysis. This technique was first described by Bizzi et al. (1990) in primates. These authors reported a range of values for $r$ : 0.71 0.81 for T2 fast spin-echo, $0.70-0.78$ for R2, 0.79-0.83 for R2*, and 0.66-0.77] for phase images. All were significant $(p<$ 0.001). Using exactly the same protocol for QSM (total variation regularization approach), Sun et al. (2015) found a range of $0.79-0.93(p<0.001)$.

Dusek et al. (2016) used a different histological technique. After digitization and color deconvolution of DAB-specific slices with ImageJ (Schindelin et al., 2012), 8-bit grayscale images were inverted and thresholded so that only gray values above 234/255 (empirically determined), representing the most intense staining, were segmented. An index representing the proportion of tissue with a positive Turnbull signal was defined as the area fraction reflecting the percentage of pixels with an intensity above 234. Values were averaged for basal ganglia ROIs. The authors found $r$ values of $0.75-0.8(p=0.01-0.02)$ with R2*, consistent with the AAS technique ( $r=0.84$ ) applied to the same sample. Wiggermann et al. (2017) used roughly the same methodology to determine area fraction, and they also determined the integrated density of iron, based on the 8-bit gray values. Although they failed to find a correlation with QSM, they focused on MS lesions, and as has already been said, this is greatly complicated by low sampling exhaustiveness and the complex relation between MS lesions, myelin and iron. When Bagnato et al. (2018) used approximately the same histological quantification method and the same iron staining in MS as Wiggerman et al, but with ROIs in different locations across the brain, they found $r$ values of 0.42 0.86 , with $\mathrm{R} 2 *$ depending on the selected region (cortex or diffuse white-matter injury vs. basal ganglia).

After digitization and binarization of Perls' images of the substantia nigra, Lee et al. $(2016,2018)$ coregistered histology/MRI images by downsampling histology images and upsampling MRI images, and then carried out a rigid transformation for a perfect match, in order to perform voxelby-voxel correlations (considering the histological images as $3 \mathrm{D}$ structures). These authors found that the $\mathrm{T} 2^{*}$ map was more closely correlated with iron $(r=-0.65--0.11)$ than the T1map, $\mathrm{T} 2 \mathrm{map}$, or $\mathrm{T} 2 * / \mathrm{T} 2$ and $\mathrm{T} 2 * / \mathrm{T}_{2}{ }^{2}$, which appeared to correlate better with neuromelanin. Surprisingly, results for SWI were in the same order of magnitude as those for the $\mathrm{T} 2 *$ map, but QSM (MEDI) results appeared less closely correlated with iron than SWI.

After digitization, Bulk et al. (2018a) eliminated background noise before downsizing histological images to MRI resolution to facilitate registration. Images were then coregistered by nonlinear transformation. ROI masks were applied and pixel-based 
spatial correlation of the signal intensities was undertaken. The authors found a correlation ( $r=0.6, p$ not available) between Perls + DAB and T2* in the frontal gyrus sample of controls.

After digitizing the images, Hametner et al. (2018) carried out color deconvolution before converting the images into 8-bit grayscale and inverting them. The histological and MR images were not coregistered. Mean intensities were measured in ROIs located in exactly the same regions on both sets of images. The authors found correlations of $r=0.62$ for R2 ${ }^{*}$ and $r=0.45$ for QSM $(p<0.0001)$. These results were poorer than those yielded by a ferrozine assay on the same specimens $(r=0.74$ for R2* and $r=0.76$ for QSM). They also plotted the iron concentration from the ferrozine assay as a function of the iron intensities from staining and found a quadratic correlation. In other words, the more intense the iron concentration, the less sensitive the staining. This could explain, at least in part, the different results for each method.

Although quantitative studies with staining are less precise than those using physical techniques, they still offer a reliable estimation of iron quantities. In addition to mapping and the possibility of visually assessing stain slices at cellular resolution, iron staining allows users to selectively stain non-heme $\mathrm{Fe}^{3+}$, $\mathrm{Fe}^{2+}$, or both. By contrast, most physical techniques quantify all the different iron forms (heme iron and non-heme $\mathrm{Fe}^{3+}$ and $\mathrm{Fe}^{2+}$ ) without distinction. However, for a good correlation, several key points have to be borne in mind, as each of these techniques can lead to biases.

\section{Limitations of ex vivo MRI, Histological Techniques and Image Coregistration Ex Vivo MRI}

Ex vivo MRI shares many sources of artifacts with in vivo MRI, but also has specific ones that it is important to be aware of before beginning any scans. First, there is the delay between the death and the MR scan, if the whole head is imaged before brain extraction, or between the death and the brain extraction. Autolysis begins as soon as death occurs, with an increase in extracellular water content, a $\mathrm{pH}$ reduction, and a reduction in temperature, leading to shorter T1 values and longer T2 ones (Dawe et al., 2009; Tashiro et al., 2015). The goal of chemical fixation is to preserve postmortem tissue in a state close to the living condition. There are many fixative agents around, but the most widely used is formaldehyde. The nature of the fixative solution affects relaxation times and diffusion properties (Shepherd et al., 2009; Birkl et al., 2018). Formaldehyde solutions can be prepared with very different concentrations, even if the most frequently used is $4 \%$, leading to $10 \%$ formalin (formaldehyde and formalin are different). This can modify the time needed for the optimum fixation of the brain tissue (Birkl et al., 2018). The volume of material to be fixed is, of course, also important. Fixation reduces T1 relaxation, T2/T2* relaxation and proton density, and increases myelin water fraction (YongHing et al., 2005; Dawe et al., 2009; Birkl et al., 2016; Shatil et al., 2018). The length of immersion in formalin is of crucial interest, as relaxation properties are not modified in the same manner if the brain specimen is fixed for a few days or for

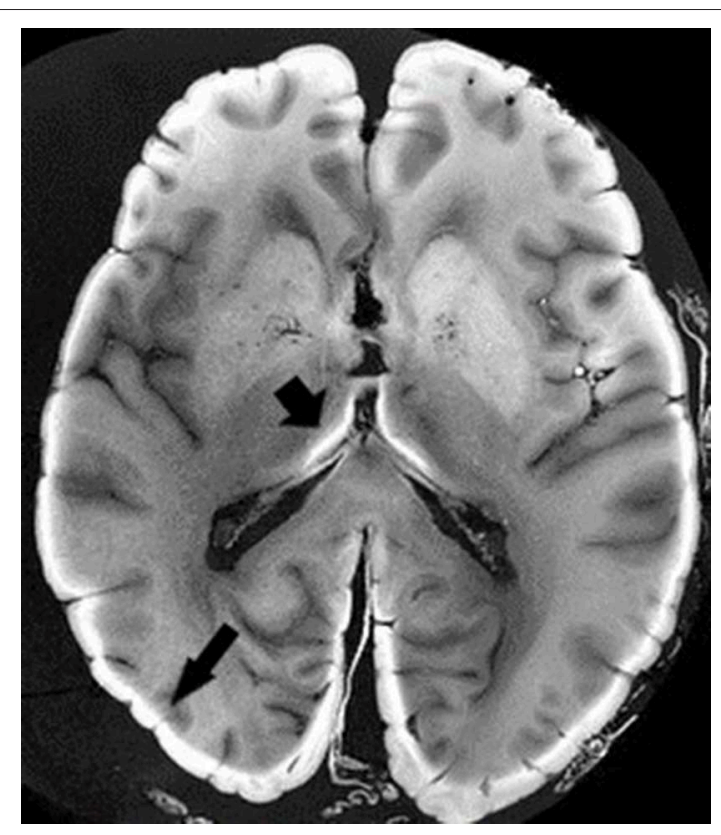

FIGURE 3 | Ex vivo MRI. T1-weighted sequence. Black arrows depict light band of formalin-fixed tissue. Image obtained during experimental procedures with all appropriate ethical approval. Included for illustrative purposes.

several years. This is due to the two-step fixation mechanism. The fixation process initially involves chemical reactions between formaldehyde and water creating methylene glycol, which rapidly penetrates brain tissue, owing to a low molecular weight, creating dehydration. This step is partly reversible by rehydration of the anatomical specimen before imaging (Shepherd et al., 2009). The second step of the fixation process leads to irreversible tissue damage by creating methylene bridges between macromolecules (Birkl et al., 2016). Some authors consider 5-6 weeks in 20\% formalin to be the optimum length of time for complete fixation, but this period is very variable, depending on the fixative agent, concentration, and volume of the specimen (Yong-Hing et al., 2005). A long fixation period (e.g., in a brain bank) leads to histological artifacts (van Duijn et al., 2011). In T1-weighted (but not T2- or proton density-weighted) images, the formalinfixed tissue appears as a light band that gradually widens over time (Figure 3). Dawe et al. (2009) analyzed differences in T2 relaxation caused by formalin fixation, comparing surface and deep brain tissue. T2 values for surface tissue rapidly decreased then plateaued, whereas for deep tissue, they rapidly decreased then slowly rose to a plateau, owing to the ongoing autolysis process. We can speculate that the first step of dehydration was responsible for the rapid decrease, and the second step of protein crosslinking was responsible for the plateauing. However, others argue that water tissue loss plays only a minor role (Birkl et al., 2016). In a recent study, QSM was carried out before death, then at weekly intervals after death for 6 weeks. The brains were in a formalin solution. Postmortem QSM correlated very closely with premortem QSM, and susceptibility remained unchanged throughout the 6 weeks (Evia et al., 2017). 


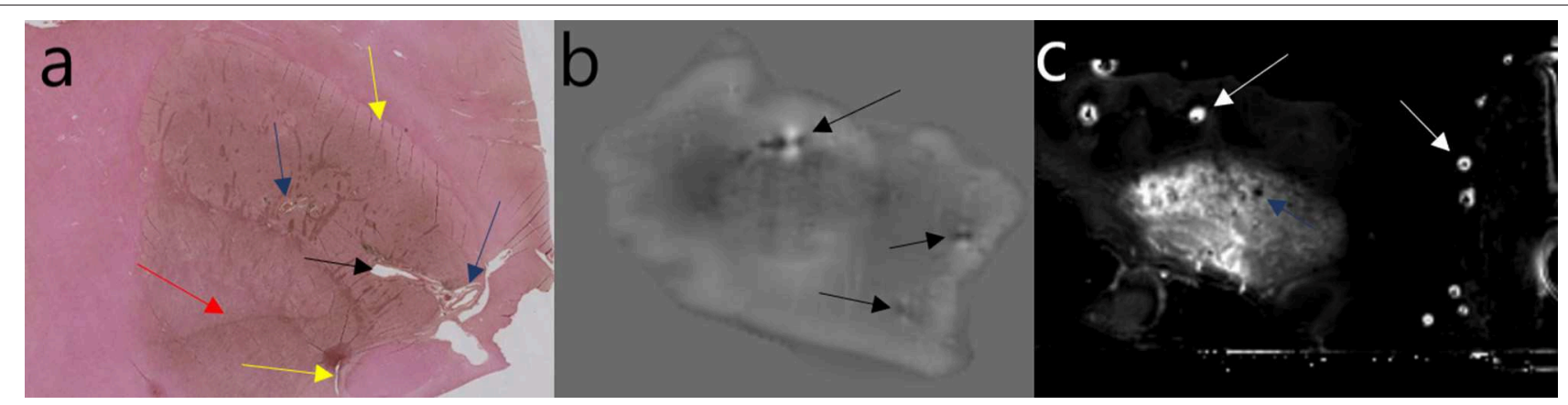

FIGURE 4 | Example of artifacts. Perls + DAB: yellow arrow = tears; black arrow = vascular dilation; blue arrow = paravascular dilation; red arrow = knife mark (a). Phase image: black arrow = vascular blood clot (b). R2* image: white arrows = trapped air bubbles; blue arrow = paravascular dilation (c). Image obtained during experimental procedures with all appropriate ethical approval. Included for illustrative purposes.

Unlike in vivo MRI, the temperature at which the scan is performed affects the signal. T1 and, to a lesser extent, T2 and T2* relaxation times decrease when the temperature drops (Birkl et al., 2016). However, as seen above, we can take advantage of this property and use the temperature coefficient as a measure of paramagnetic susceptibility (Birkl et al., 2015, 2017).

Concerning rehydration periods before MRI, our literature review showed that some authors only immersed the specimen in saline solution just before scan, whereas others soaked the specimen for $12,24 \mathrm{~h}$ or more, changing the solution several times (Shepherd et al., 2009; De Reuck et al., 2011; Blazejewska et al., 2013; Wallace et al., 2016).

The medium in which the brain is imaged is also of particular importance, as it can affect the signal and disturb susceptibility values. Proton-free fluorinated fluids are regarded as the gold standard, as they are inert, produce no MRI signal, and have a similar magnetic susceptibility to tissue (Miller et al., 2011; Shatil et al., 2016). However, availability, cost, and possible interferences with subsequent histology limit their use. Some authors use a solid (paraffin/plastic polymer mixture) or semisolid (agarose, gelatin) medium, to avoid vibrations, but very little is known about their contribution to the MR signal. The present review revealed the use of many different protocols, some involving fluorinated fluids and others directly a formalin or saline solution, while many teams used a solid or semisolid medium.

Four important sources of artifacts with ex vivo MRI are air bubbles, intravascular blood clots, paravascular space dilation, and vibrations in the scanner. The air/tissue interface induces susceptibility artifacts that can seriously compromise the interpretation of susceptibility-based sequences. A liquid medium allows air bubbles to be eliminated by complete immersion and gentle shaking of the container. However, if the anatomical specimen is not correctly embedded (e.g., with gauze), it is exposed to vibrations, yielding uninterpretable images. A semisolid or solid medium often allows vibrations to be avoided, but managing air bubbles is much more complicated and requires considerable experience (Shatil et al., 2016). Another solution is to image the whole head, as many teams did with fresh specimens, but to our knowledge, nobody has yet tried to image a fixed whole head in order to examine iron. Intravascular blood clots can be limited by washing the vessels in the forensic or anatomy laboratory. When the specimen is stored for a long time before imaging, blood seems to dilute in the fixative solution. Paravascular enlargement can be seen in vivo (Virchow-Robin space) (Bacyinski et al., 2017), and when the brain is fixed before imaging, the shrinkage seems to accentuate this (Figure 4). It can also be seen in histological studies.

Specimen orientation in the scanner is a theoretical problem for ex vivo MRI compared with in vivo MRI, especially with small anatomical specimens. It is important to think about this before the scan, in order to avoid having to reorient the histological sections during image post-processing, which is a potential source of image information loss. Furthermore, with small specimens, it can be hard to find an acquisition plane that is commonly used for in vivo MRI, making it more difficult to extrapolate from the results. Once again, a rigorous methodology is essential.

In contrast to in vivo MRI, whole brains or small parts of the brain can be imaged using a wide variety of radiofrequency coils. Dedicated homebuilt coils can be designed, or dedicated coils for other anatomical body parts (e.g., wrist) can be diverted, in order to achieve a better signal/noise ratio and a better contrast. However, this can impinge on the comparability of results across centers.

The present review showed that scanner protocols for ironbased sequences can differ greatly across studies. The main differences we found lay in the total scan duration, which could range from $2 \mathrm{~min}$ to more than $6 \mathrm{~h}$ for quantitative studies with a high variability (Table 3 ), and be very much longer still for other studies. Spatial resolution and slice thickness mainly depended on the scanner's capacities, but it is interesting to note how they varied across studies. The number of signals averaged also differed across studies, as did the number of echoes performed during a multi-gradient echo sequence for $\mathrm{T}^{*}$ and $\mathrm{R} 2 *$. Not only the number of echoes, but also the interecho time and the range of this echo time, are important to precisely describe R2*(Péran et al., 2007). QSM can be calculated with single-echo gradient acquisition, but multi-echo gradient acquisition ensures a better signal/noise ratio (Deistung et al., 2016). With QSM, the multitude of post-processing algorithms available can lead to 
TABLE 5 | Key points to consider in histology, ex vivo MRI, iron-based sequences and image coregistration, in order to limit methodological problems and to compare studies.

\begin{tabular}{|c|c|c|c|}
\hline Ex vivo MRI & Iron-based sequences & Histology & Image coregistration \\
\hline Time between death and MRI (fresh) & Spatial resolution & Slice thickness & Image file formats \\
\hline Time between death and fixation (fixed) & Slice thickness & Staining protocol & Up- or downsampling image resolution \\
\hline Fixation period before MRI & Acquisition time & Type of staining & Non-linear transformation? \\
\hline Fixative agent & NSA & Fixation time between MRI and histology & Stack procedure? \\
\hline Degree of putrefaction & Number of echoes (relaxometry) & Tears & Color deconvolution? \\
\hline Imaging solution & Echo time (relaxometry) & Stretching & Thresholded out background \\
\hline Rehydration or not (fixed) & Post-processing algorithms (QSM) & Shrinkage & Grayscale conversion \\
\hline Rehydration time (fixed) & & Distortions & \\
\hline Room temperature & & Swelling & \\
\hline Air/tissue interface (bubbles) & & Blood vessel or paravascular space dilation & \\
\hline Contention technique to avoid vibrations & & Knife mark & \\
\hline Specimen orientation & & Sampling & \\
\hline \multicolumn{4}{|l|}{ RF coil type } \\
\hline $\begin{array}{l}\text { Intravascular blood clot or paravascular } \\
\text { space dilation }\end{array}$ & & & \\
\hline
\end{tabular}

NSA, number of signals averaged; QSM, quantitative susceptibility mapping; RF, radiofrequency; ROI, region of interest.

slightly different results (Langkammer et al., 2017). Once again, we need to be cautious when comparing different studies with different QSM or R2/R2* protocols (especially if the histological protocol also differs) (see Table 5).

\section{Histological Techniques}

MRI has its fair share of random variables, but histology is also a source of limitations that need to be borne in mind. As we can see in Table 4, slice thickness varied greatly across studies, ranging from $5 \mu \mathrm{m}$ to $8 \mathrm{~mm}$. It is not important when a simple visual assessment is carried out, but the determination of iron intensities may vary according to the thickness of the stain slice. Most of the time, histological slices are several micrometers thick, whereas MRI slices are hundred micrometers for the best resolution, raising questions about the comparability of results. At the micrometer scale, not all the slices can be stained, and sampling is mandatory (e.g., 1 in every 20 slices stained for example), reducing the representativeness of the data. Conversely, some authors used a thickness of $8 \mathrm{~mm}$, with an MRI slice thickness of $2 \mathrm{~mm}$ (Walsh et al., 2013; Sun et al., 2015). The intensity of the stained slices may not reflect the MRI signal intensity measure. Along with slice thickness, stain protocols differ greatly across studies, even if we only consider a single staining technique such as Perls' (see Table 4). It should, however, be noted that the Turnbull + DAB procedure after ammonium sulfide seemed to be the standard in many recent studies. The choice of staining procedure (Perls', Turnbull, ferritin immunohistochemistry) and the application (or not) of DAB can make a considerable difference (van Duijn et al., 2013).

Time between MRI and the staining procedure is important, as iron leakage in a formalin solution has been shown to increase over time, leading to correlation difficulties (Gellein et al., 2008; Schrag et al., 2010a; Krebs et al., 2014). However, significant differences only appear for very long-term storage, and are almost negligible for the first 12 weeks.
The last important point concerns histological processing artifacts. Fixation, inclusion, cutting and staining can lead to distortions, tears, shrinkage, swelling, stretching, blade marks, and so on that can affect the correlation process (Figure 4). To avoid these technical limitations, some authors recommend using thick histological sections (Alho et al., 2017). This is an interesting method, for if the main goal of these authors is to perform perfect matching without a quantitative analysis, then homogenizing the slice thicknesses for MRI and histology can presumably also ameliorate quantitative correlations (see Table 5).

\section{Image Coregistration}

Technological advances now allow histological images to be digitized, and thus to be matched with MR images. A great many processes are needed, involving downsampling, linear and nonlinear transformations, in order to perfectly match the two sets of images. Another possible method is to stack the histological images to create a $3 \mathrm{D}$ volume that can be matched with 3D-MRI volume. These procedures are often used by creating an MRI-histology-based atlas (Yelnik et al., 2007; Choe et al., 2011; Adler et al., 2014), but all this image post-processing raises questions about a possible loss of information between the raw data and the final image, even if images are stored in a format with no data compression. We can ask the same questions about the color deconvolution algorithms and grayscale conversion used by some authors in the present review. Thresholding the background in order to keep only certain information is often empirical, according to some authors (Dusek et al., 2016), and can vary from one team to another (see Table 5).

\section{Is There an Ideal Coregistration Method?}

Given the published literature, we think the simpler the method is, the better it is for final coregistration. The first step is to image your brain sample with minimal artifacts. Unfortunately, there 
is no perfect method and the best is to experiment for oneself, learning from errors and successes. The second step is to clearly define what is the goal of your histology. Is it to match perfectly the two sets of images or to perform quantitative measures? Often it is necessary to find a good compromise. During histological process, it is important to never forget the final coregistration process in order to limit in the last step (coregistration) as much as possible the need for image manipulation (color deconvolution, downsizing, image transformation...). We would like to give you an ideal standardized method but due to all method possibilities listed above, there is not a perfect way to do and each team have to experiment by oneself with its own available resources.

\section{CONCLUSION}

We reviewed all the relevant literature concerning ex vivo MRI and iron histology and came to three main findings. First, matching ex vivo MRI with histology allows for a better understanding of the physiopathology of some neurological disorders in which iron is suspected to play a role, as well as of the radiological anatomy of specific neurological structures. Second, correlating iron-based MRI sequences with iron quantification performed with a reference physical technique shows that MRI sequences can be reliably used for the in vivo estimation of iron in the brain. It is interesting to note that results from ex vivo MRI were in accordance with in vivo data correlated using

\section{REFERENCES}

Adler, D. H., Pluta, J., Kadivar, S., Craige, C., Gee, J. C., Avants, B. B., et al. (2014). Histology-derived volumetric annotation of the human hippocampal subfields in postmortem MRI. NeuroImage. JANV 84, 505-523. doi: 10.1016/j.neuroimage.2013.08.067

Alho, E. J. L., Alho, A. T. D. L., Grinberg, L., Amaro, E., Dos Santos, G. A. B., da Silva, R. E., et al. (2017). High thickness histological sections as alternative to study the three-dimensional microscopic human sub-cortical neuroanatomy. Brain Struct. Funct 223, 1121-1132. doi: 10.1007/s00429-017-1548-2

Antharam, V., Collingwood, J. F., Bullivant, J.-P., Davidson, M. R., Chandra, S., Mikhaylova, A., et al. (2012). High field magnetic resonance microscopy of the human hippocampus in Alzheimer's disease: quantitative imaging and correlation with iron. Neuroimage 59, 1249-1260. doi: 10.1016/j.neuroimage.2011.08.019

Ashraf, A., Clark, M., and So, P. -W. (2018). The aging of iron man. Front. Aging Neurosci. 10:65. doi: 10.3389/fnagi.2018.00065

Bacyinski, A., Xu, M., Wang, W., and Hu, J. (2017). The paravascular pathway for brain waste clearance: current understanding, significance and controversy. Front. Neuroanat. 11:00101. doi: 10.3389/fnana.2017.00101

Bagnato, F., Hametner, S., Boyd, E., Endmayr, V., Shi, Y., Ikonomidou, V., et al. (2018). Untangling the $\mathrm{R}^{*}$ contrast in multiple sclerosis: a combined MRI-histology study at 7.0 tesla. PLoS ONE 13:e0193839. doi: 10.1371/journal.pone.0193839

Bagnato, F., Hametner, S., Yao, B., van Gelderen, P., Merkle, H., Cantor, F. K., et al. (2011). Tracking iron in multiple sclerosis: a combined imaging and histopathological study at 7 tesla. Brain 134, 3599-3612. doi: 10.1093/brain/awr278

Barbagallo, G., Sierra-Peña, M., Nemmi, F., Traon, A. P., Meissner, W. G., Rascol, O., et al. (2016). Multimodal MRI assessment of nigro-striatal pathway in multiple system atrophy and Parkinson disease. Mov. Disord. 31, 325-334. doi: $10.1002 / \mathrm{mds} .26471$
Hallgren and Sourander's equations. Third and last, data from ex vivo MRI correlated with quantitative staining seem to be a good approximation of the results yielded by physical techniques, and histology has the added advantages of being relatively inexpensive and offering opportunities for mapping. However, matching ex vivo MRI and histology raises some methodological issues that need to be taken into account before extrapolating to in vivo MRI.

\section{DATA AVAILABILITY}

No datasets were generated or analyzed for this study.

\section{AUTHOR CONTRIBUTIONS}

$\mathrm{AD}, \mathrm{GA}$, and PP contributed conception and design of the review. $\mathrm{AD}$ and JC wrote the first draft of the manuscript. GA wrote sections of the manuscript. AD, GA, and JC collected data. PC reviewed specifically histological and anatomical parts. PP reviewed specifically MRI parts. All authors contributed to manuscript revision, read and approved the submitted version.

\section{FUNDING}

Funding was supported by Fondation pour la recherche médicale (FRM); Grant numbers: DEA20170839104. It was the sole funding.
Bartzokis, G., Tishler, T. A., Lu, P. H., Villablanca, P., Altshuler, L. L., Carter, M., et al. (2007). Brain ferritin iron may influence age- and gender-related risks of neurodegeneration. Neurobiol. Aging 28, 414-423. doi: 10.1016/j.neurobiolaging.2006.02.005

Bennett, K. M., Shapiro, E. M., Sotak, C. H., and Koretsky, A. P. (2008). Controlled aggregation of ferritin to modulate MRI relaxivity. Biophys. J. 95, 342-351. doi: 10.1529/biophysj.107.116145

Bilgic, B., Pfefferbaum, A., Rohlfing, T., Sullivan, E. V., and Adalsteinsson, E. (2012). MRI estimates of brain iron concentration in normal aging using quantitative susceptibility mapping. Neuroimage 59, 2625-2635. doi: 10.1016/j.neuroimage.2011.08.077

Birkl, C., Carassiti, D., Hussain, F., Langkammer, C., Enzinger, C., Fazekas, F., et al. (2017). Assessment of ferritin content in multiple sclerosis brains using temperature-induced R*2changes. Magn. Reson. Med. 79, 1609-1615. doi: $10.1002 / \mathrm{mrm} .26780$

Birkl, C., Langkammer, C., Golob-Schwarzl, N., Leoni, M., Haybaeck, J., Goessler, W., et al. (2016). Effects of formalin fixation and temperature on MR relaxation times in the human brain. NMR Biomed. 29, 458-465. doi: 10.1002/nbm.3477

Birkl, C., Langkammer, C., Krenn, H., Goessler, W., Ernst, C., Haybaeck, J., et al. (2015). Iron mapping using the temperature dependency of the magnetic susceptibility. Magn. Reson. Med. 73, 1282-1288. doi: 10.1002/mrm.25236

Birkl, C., Soellradl, M., Toeglhofer, A. M., Krassnig, S., Leoni, M., Pirpamer, L., et al. (2018). Effects of concentration and vendor specific composition of formalin on postmortem MRI of the human brain. Magn. Reson. Med. 79, 1111-1115. doi: 10.1002/mrm.26699

Bizzi, A., Brooks, R. A., Brunetti, A., Hill, J. M., Alger, J. R., Miletich, R. S., et al. (1990). Role of iron and ferritin in MR imaging of the brain: a study in primates at different field strengths. Radiology 177, 59-65. doi: 10.1148/radiology.177.1.2399339

Blazejewska, A. I., Schwarz, S. T., Pitiot, A., Stephenson, M. C., Lowe, J., Bajaj, N., et al. (2013). Visualization of nigrosome 1 and its loss in PD. Neurology 81, 534-540. doi: 10.1212/WNL.0b013e31829e6fd2 
Bossoni, L., Grand Moursel, L., Bulk, M., Simon, B. G., Webb, A., van der Weerd, L., et al. (2017). Human-brain ferritin studied by muon spin rotation: a pilot study. J. Phys. Condens Matter. 29:415801. doi: 10.1088/1361-648X/aa80b3

Bulk, M., Abdelmoula, W. M., Nabuurs, R. J. A., van der Graaf, L. M., Mulders, C. W. H., Mulder, A. A., et al. (2018a). Postmortem, M. R. I., and histology demonstrate differential iron accumulation and cortical myelin organization in early- and late-onset Alzheimer's disease. Neurobiol. Aging 62, 231-242. doi: 10.1016/j.neurobiolaging.2017.10.017

Bulk, M., van der Weerd, L., Breimer, W., Lebedev, N., Webb, A., Goeman, J. J., et al. (2018b). Quantitative comparison of different iron forms in the temporal cortex of Alzheimer patients and control subjects. Sci. Rep. 8:6898. doi: 10.1038/s41598-018-25021-7

Bush, W. D., Garguilo, J., Zucca, F. A., Bellei, C., Nemanich, R. J., Edwards, G. S., et al. (2009). Neuromelanins isolated from different regions of the human brain exhibit a common surface photoionization threshold. Photochem. Photobiol. 85, 387-390. doi: 10.1111/j.1751-1097.2008.00476.x

Chen, J. C., Hardy, P. A., Clauberg, M., Joshi, J. G., Parravano, J., Deck, J. H., et al. (1989). T2 values in the human brain: comparison with quantitative assays of iron and ferritin. Radiology 173, 521-526. doi: 10.1148/radiology.173.2.2798884

Choe, A. S., Gao, Y., Li, X., Compton, K. B., Stepniewska, I., and Anderson, A. W. (2011). Accuracy of image registration between MRI and light microscopy in the ex vivo brain. Magn. Reson. Imag. 29, 683-692. doi: 10.1016/j.mri.2011.02.022

Connor, J. R., Snyder, B. S., Arosio, P., Loeffler, D. A., and LeWitt, P. (1995). A quantitative analysis of isoferritins in select regions of aged, parkinsonian, and Alzheimer's diseased brains. J. Neurochem. 65, 717-724. doi: 10.1046/j.1471-4159.1995.65020717.x

Dal-Bianco, A., Grabner, G., Kronnerwetter, C., Weber, M., Höftberger, R., Berger, T., et al. (2017). Slow expansion of multiple sclerosis iron rim lesions: pathology and $7 \mathrm{~T}$ magnetic resonance imaging. Acta Neuropathol. 133, 25-42. doi: 10.1007/s00401-016-1636-Z

Daou, T. (2007). Synthèse et fonctionnalisation de nanoparticules d'oxydes de fer magnétiques [Dissertation]. Strasbourg: Louis Pasteur University.

Dawe, R. J., Bennett, D. A., Schneider, J. A., Vasireddi, S. K., and Arfanakis, K. (2009). Postmortem MRI of human brain hemispheres: T2 relaxation times during formaldehyde fixation. Magn. Reson. Med. 61, 810-818. doi: 10.1002/mrm.21909

de Hollander, G., Keuken, M. C., Bazin, P.-L., Weiss, M., Neumann, J., Reimann, K., et al. (2014). A gradual increase of iron toward the medialinferior tip of the subthalamic nucleus. Hum. Brain Mapp. 35, 4440-4449. doi: 10.1002/hbm.22485

De Reuck, J., Auger, F., Cordonnier, C., Deramecourt, V., Durieux, N., Pasquier, F., et al. (2011). Comparison of 7.0-T T2*-magnetic resonance imaging of cerebral bleeds in post-mortem brain sections of Alzheimer patients with their neuropathological correlates. Cerebrovasc. Dis. 31, 511-517. doi: 10.1159/000324391

De Reuck, J., Devos, D., Moreau, C., Auger, F., Durieux, N., Deramecourt, V., et al. (2017). Topographic distribution of brain iron deposition and small cerebrovascular lesions in amyotrophic lateral sclerosis and in frontotemporal lobar degeneration: a post-mortem 7.0-tesla magnetic resonance imaging study with neuropathological correlates. Acta Neurol. Belg. 117, 873-878. doi: 10.1007/s13760-017-0832-5

de Rochefort, L., et al. (2010). Quantitative susceptibility map reconstruction from MR Phase data using Bayesian regularization: validation and application to brain imaging. Magn. Reson. Med. 63, 194- 206. doi: 10.1002/mrm.22187

Deistung, A., Schweser, F., and Reichenbach, J. R. (2016). Overview of quantitative susceptibility mapping. NMR Biomed. 30:e3569. doi: 10.1002/nbm.3569

Dhenain, M., Privat, N., Duyckaerts, C., and Jacobs, R. E. (2002). Senile plaques do not induce susceptibility effects in T2*-weighted MR microscopic images. NMR Biomed. 15, 197-203. doi: 10.1002/nbm.760

Dormont, D., Ricciardi, K. G., Tandé, D., Parain, K., Menuel, C., Galanaud, D., et al. (2004). Is the subthalamic nucleus hypointense on T2-weighted images? A correlation study using MR imaging and stereotactic atlas data. Am. J. Neuroradiol. 25, 1516-1523.

Double, K. L., Dedov, V. N., Fedorow, H., Kettle, E., Halliday, G. M., Garner, B., et al. (2008). The comparative biology of neuromelanin and lipofuscin in the human brain. Cell Mol. Life Sci. 65, 1669-1682. doi: 10.1007/s00018-00 8-7581-9
Dusek, P., Bahn, E., Litwin, T., Jabłonka-Salach, K., Łuciuk, A., Huelnhagen, T., et al. (2016). Brain iron accumulation in Wilson disease: a post-mortem 7 tesla MRI - histopathological study. Neuropathol. Appl. Neurobiol. 43, 514-532. doi: $10.1111 /$ nan. 12341

Dusek, P., Jankovic, J., and Le, W. (2012). Iron dysregulation in movement disorders. Neurobiol. Dis. 46, 1-18. doi: 10.1016/j.nbd.2011.12.054

Dusek, P., Roos, P. M., Litwin, T., Schneider, S. A., Flaten, T. P., and Aaseth, J. (2015). The neurotoxicity of iron, copper and manganese in Parkinson's and Wilson's diseases. J. Trace Elements Med. Biol. 31, 193-203. doi: 10.1016/j.jtemb.2014.05.007

Engelen, M., Vanna, R., Bellei, C., Zucca, F. A., Wakamatsu, K., Monzani, E., et al. (2012). Neuromelanins of human brain have soluble and insoluble components with dolichols attached to the melanic structure. PLoS ONE 7:e48490. doi: 10.1371/journal.pone.0048490

Evia, A. M., Kotrotsou, A., Tamhane, A. A., Dawe, R. J., Kapasi, A., Leurgans, S. E., et al. (2017). Ex-vivo quantitative susceptibility mapping of human brain hemispheres. PLoS ONE 12:0188395. doi: 10.1371/journal.pone.0188395

Faucheux, B. A., Martin, M.-E., Beaumont, C., Hauw, J.-J., Agid, Y., and Hirsch, E. C. (2003). Neuromelanin associated redox-active iron is increased in the substantia nigra of patients with Parkinson's disease. J. Neurochem. 86, 1142-1148. doi: 10.1046/j.1471-4159.2003.01923.x

Foroutan, P., Murray, M. E., Fujioka, S., Schweitzer, K. J., Dickson, D. W., Wszolek, Z. K., et al. (2013). Progressive supranuclear palsy: high-field-strength MR microscopy in the human substantia nigra and globus pallidus. Radiology 266, 280-288. doi: 10.1148/radiol.12102273

Fujii, S., Matsusue, E., Kinoshita, T., Sugihara, S., Ohama, E., and Ogawa, T. (2007). Hyperintense putaminal rim at $3 \mathrm{~T}$ reflects fewer ferritin deposits in the lateral marginal area of the putamen. Am. J. Neuroradiol. 28, 777-781.

Fukunaga, M., Li, T.-Q., van Gelderen, P., de Zwart, J. A., Shmueli, K., Yao, B., et al. (2010). Layer-specific variation of iron content in cerebral cortex as a source of MRI contrast. Proc. Natl. Acad. Sci. U.S.A. 107, 3834-3839. doi: 10.1073/pnas.0911177107

Gellein, K., Flaten, T. P., Erikson, K. M., Aschner, M., and Syversen, T. (2008). Leaching of trace elements from biological tissue by formalin fixation. Biol. Trace Elem. Res. 121, 221-225. doi: 10.1007/s12011-007-8051-1

Ghassaban, K., Liu, S., Jiang, C., and Haacke, E. M. (2018). Quantifying iron content in magnetic resonance imaging. Neuroimage 187, 77-92. doi: 10.1016/j.neuroimage.2018.04.047

Haacke, E. M., Cheng, N. Y., House, M. J., Liu, Q., Neelavalli, J., Ogg, R. J., et al. (2005). Imaging iron stores in the brain using magnetic resonance imaging. Magn. Reson. Imag. 23, 1-25. doi: 10.1016/j.mri.2004.10.001

HALLGREN, B., and SOURANDER, P. (1958). The effect of age on the non-haemin iron in the human brain. J. Neurochem. 3, 41-51. doi: 10.1111/j.1471-4159.1958.tb12607.x

Hametner, S., Endmayr, V., Deistung, A., Palmrich, P., Prihoda, M., Haimburger, E., et al. (2018). The influence of brain iron and myelin on magnetic susceptibility and effective transverse relaxation - a biochemical and histological validation study. Neuroimage 179, 117-133. doi: 10.1016/j.neuroimage.2018.06.007

Hansen, A. K., Knudsen, K., Lillethorup, T. P., Landau, A. M., Parbo, P., Fedorova, T., et al. (2016). In vivo imaging of neuromelanin in Parkinson's disease using 18F-AV-1451 PET. Brain 139(Pt 7):2039-2049. doi: 10.1093/brain/aww098

Hare, D. J., Ayton, S., Bush, A., and Lei, P. (2013). A delicate balance: iron metabolism and diseases of the brain. Front. Aging Neurosci. 5:34. doi: 10.3389/fnagi.2013.00034

Harrison, P. M., and Arosio, P. (1996). The ferritins: molecular properties, iron storage function and cellular regulation. Biochim. Biophys. Acta Bioenergetics 1275, 161-203. doi: 10.1016/0005-2728(96)00022-9

Hopp, K., Popescu, B. F., McCrea, R. P., Harder, S. L., Robinson, C. A., Haacke, M. E., et al. (2010). Brain iron detected by SWI high pass filtered phase calibrated with synchrotron X-ray fluorescence. J. Magn. Reson. Imaging 31, 1346-1354. doi: 10.1002/jmri.22201

House, M. J., St. Pierre, T. G., Kowdley, K. V., Montine, T., Connor, J., Beard, J., et al. (2007). Correlation of proton transverse relaxation rates (R2) with iron concentrations in postmortem brain tissue from Alzheimer's disease patients. Magn. Reson. Med. 57, 172-180. doi: 10.1002/mrm.21118

House, M. J., St. Pierre, T. G., and McLean, C. (2008). 1.4T study of proton magnetic relaxation rates, iron concentrations, and plaque burden in 
Alzheimer's disease and control postmortem brain tissue. Magn. Reson. Med. 60, 41-52. doi: 10.1002/mrm.21586

Jiang, H., Wang, J., Rogers, J., and Xie, J. (2017). Brain iron metabolism dysfunction in Parkinson's disease. Mol. Neurobiol. 54, 3078-3101. doi: 10.1007/s12035-016-9879-1

Kaunzner, U. W., Kang, Y., Zhang, S., Morris, E., Yao, Y., Pandya, S., et al. (2019). Quantitative susceptibility mapping identifies inflammation in a subset of chronic multiple sclerosis lesions. Brain 142, 133-145. doi: 10.1093/brain/awy296

Koeppen, A. H. (1995). The history of iron in the brain. J. Neurol. Sci. 134, 1-9. doi: 10.1016/0022-510X(95)00202-D

Krebs, N., Langkammer, C., Goessler, W., Ropele, S., Fazekas, F., Yen, K., et al. (2014). Assessment of trace elements in human brain using inductively coupled plasma mass spectrometry. J. Trace. Elem. Med. Biol. 28, 1-7. doi: 10.1016/j.jtemb.2013.09.006

Kumar, P., Bulk, M., Webb, A., van der Weerd, L., Oosterkamp, T. H., Huber, M., et al. (2016). A novel approach to quantify different iron forms in ex-vivo human brain tissue. Sci. Rep. 6:38916. doi: 10.1038/srep38916

Kwan, J. Y., Jeong, S. Y., Van Gelderen, P., Deng, H.-X., Quezado, M. M., Danielian, L. E., et al. (2012). Iron accumulation in deep cortical layers accounts for MRI signal abnormalities in ALS: correlating 7 tesla MRI and pathology. PLoS ONE 7:0035241. doi: 10.1371/journal.pone.0035241

Langkammer, C. (2012). Susceptibility induced gray-white matter MRI contrast in the human brain. Neuroimage 59, 1413-1419. doi: 10.1016/j.neuroimage.2011.08.045

Langkammer, C., Krebs, N., Goessler, W., Scheurer, E., Ebner, F., Yen, K., et al. (2010). Quantitative MR imaging of brain iron: a postmortem validation study. Radiology 257, 455-462. doi: 10.1148/radiol.10100495

Langkammer, C., Schweser, F., Krebs, N., Deistung, A., Goessler, W., Scheurer, E., et al. (2012). Quantitative susceptibility mapping (QSM) as a means to measure brain iron? A post mortem validation study. Neuroimage 62:1593-9. doi: 10.1016/j.neuroimage.2012.05.049

Langkammer, C., Schweser, F., Shmueli, K., Kames, C., Li, X., Guo, L., et al. (2017). Quantitative susceptibility mapping: report from the 2016 reconstruction challenge. Magn. Reson. Med. 79, 1661-1673. doi: 10.1002/mrm.26830

Lee, H., Baek, S.-Y., Chun, S. Y., Lee, J.-H., and Cho, H. (2018). Specific visualization of neuromelanin-iron complex and ferric iron in the human post-mortem substantia nigra using MR relaxometry at 7T. Neuroimage 172, 874-885. doi: 10.1016/j.neuroimage.2017.11.035

Lee, J.-H., Baek, S.-Y., Song, Y., Lim, S., Lee, H., Nguyen, M. P., et al. (2016). The neuromelanin-related $\mathrm{T} 2$ * contrast in postmortem human substantia nigra with 7T MRI. Sci. Rep. 6:32647. doi: 10.1038/srep32647

Liem, M. K., Lesnik Oberstein, S. A., Versluis, M. J., Maat-Schieman, M. L., Haan, J., Webb, A. G., et al. (2012). 7 T MRI reveals diffuse iron deposition in putamen and caudate nucleus in CADASIL. J. Neurol. Neurosurg. Psychiatry 83, 1180-1185. doi: 10.1136/jnnp-2012-302545

Massey, L. A., Miranda, M. A., Al-Helli, O., Parkes, H. G., Thornton, J. S., So, P.-W., et al. (2017). 9.4 T MR microscopy of the substantia nigra with pathological validation in controls and disease. Neuroimage Clin. 13, 154-163. doi: 10.1016/j.nicl.2016.11.015

Massey, L. A., Miranda, M. A., Zrinzo, L., Al-Helli, O., Parkes, H. G., Thornton, J. S., et al. (2012). High resolution MR anatomy of the subthalamic nucleus: imaging at 9.4T with histological validation. Neuroimage 59, 2035-2044. doi: 10.1016/j.neuroimage.2011.10.016

Matsuda, K. M., Lopes-Calcas, A., Honke, M. L., O’Brien-Moran, Z., Buist, R., West, M., et al. (2017). Ex vivo tissue imaging for radiologypathology correlation: a pilot study with a small bore 7 -T MRI in a rare pigmented ganglioglioma exhibiting complex MR signal characteristics associated with melanin and hemosiderin. JMI, JMIOBU 4:036001. doi: 10.1117/1.JMI.4.3.036001

Matsusue, E., Fujii, S., Kanasaki, Y., Kaminou, T., Ohama, E., and Ogawa, T. (2009). Cerebellar lesions in multiple system atrophy: postmortem MR imaging-pathologic correlations. Am. J. Neuroradiol. 30, 1725-1730. doi: 10.3174/ajnr.A1662

Matsusue, E., Fujii, S., Kanasaki, Y., Sugihara, S., Miyata, H., Ohama, E., et al. (2008). Putaminal lesion in multiple system atrophy: postmortem MR-pathological correlations. Neuroradiology 50, 559-567. doi: 10.1007/s00234-008-0381-y
Matusch, A., Bauer, A., and Becker, J. S. (2011). Element imaging in formalin fixed slices of human mesencephalon. Int. J. Mass Spectr. 307(1-3):240-244. doi: 10.1016/j.ijms.2011.03.006

Meadowcroft, M. D., Peters, D. G., Dewal, R. P., Connor, J. R., and Yang, Q. X. (2015). The effect of iron in MRI and transverse relaxation of amyloid-beta plaques in Alzheimer's disease. NMR Biomed. 28, 297-305. doi: $10.1002 / \mathrm{nbm} .3247$

Meguro, R., Asano, Y., Odagiri, S., Li, C., Iwatsuki, H., and Shoumura, K. (2007). Nonheme-iron histochemistry for light and electron microscopy: a historical, theoretical and technical review. Arch. Histol. Cytol. 70, 1-19. doi: 10.1679/aohc.70.1

Miller, K. L., Stagg, C. J., Douaud, G., Jbabdi, S., Smith, S. M., Behrens, T. E. J., et al. (2011). Diffusion imaging of whole, post-mortem human brains on a clinical MRI scanner. Neuroimage 57(1-4):167-81. doi: 10.1016/j.neuroimage.2011.03.070

Moher, D., Liberati, A., Tetzlaff, J., and Altman, D. G. (2009). Preferred reporting items for systematic reviews and meta-analyses: the PRISMA statement. PLoS Med. 6:1000097. doi: 10.1371/journal.pmed.1000097

Péran, P., Barbagallo, G., Nemmi, F., Sierra, M., Galitzky, M., and Traon, A. P, et al. (2018). MRI supervised and unsupervised classification of Parkinson's disease and multiple system atrophy. Mov. Disord. 33, 600-608. doi: $10.1002 / \mathrm{mds} .27307$

Péran, P., Cherubini, A., Assogna, F., Piras, F., Quattrocchi, C., Peppe, A., et al. (2010). Magnetic resonance imaging markers of Parkinson's disease nigrostriatal signature. Brain 133, 3423-3433. doi: 10.1093/brain/awq212

Péran, P., Cherubini, A., Luccichenti, G., Hagberg, G., Démonet, J.-F., Rascol, O., et al. (2009). Volume and iron content in basal ganglia and thalamus. Hum. Brain Mapp. 30, 2667-2675. doi: 10.1002/hbm.20698

Péran, P., Hagberg, G., Luccichenti, G., Cherubini, A., Brainovich, V., Celsis, P., et al. (2007). Voxel-based analysis of R2* maps in the healthy human brain. J. Magn. Reson. Imag. 26, 1413-1420. doi: 10.1002/jmri.21204

Quintana, C., Bellefqih, S., Laval, J. Y., Guerquin-Kern, J. L., Wu, T. D., Avila, J., et al. (2006). Study of the localization of iron, ferritin, and hemosiderin in Alzheimer's disease hippocampus by analytical microscopy at the subcellular level. J. Struct. Biol. 153, 42-54. doi: 10.1016/j.jsb.2005.11.001

Sasaki, M., Shibata, E., Kudo, K., and Tohyama, K. (2008). Neuromelanin-sensitive MRI. Clin. Neuroradiol. 18, 147-153. doi: 10.1007/s00062-008-8018-4

Sasaki, M., Shibata, E., Tohyama, K., Takahashi, J., Otsuka, K., Tsuchiya, K., et al. (2006). Neuromelanin magnetic resonance imaging of locus ceruleus and substantia nigra in Parkinson's disease. Neuroreport 17, 1215-1218. doi: 10.1097/01.wnr.0000227984.84927.a7

Schenck, J. F. (2003). Magnetic resonance imaging of brain iron. J Neurol Sci. 207(1-2):99-102. doi: 10.1016/S0022-510X(02)00431-8

Schindelin, J., Arganda-Carreras, I., Frise, E., Kaynig, V., Longair, M., Pietzsch, T., et al. (2012). Fiji: an open-source platform for biological-image analysis. Nat. Methods 9, 676-682. doi: 10.1038/nmeth.2019

Schrag, M., Dickson, A., Jiffry, A., Kirsch, D., Vinters, H. V., and Kirsch, W. (2010a). The effect of formalin fixation on the levels of brain transition metals in archived samples. Biometals 23, 1123-1127. doi: 10.1007/s10534-010-9359-4

Schrag, M., McAuley, G., Pomakian, J., Jiffry, A., Tung, S., Mueller, C., et al. (2010b). Correlation of hypointensities in susceptibility-weighted images to tissue histology in dementia patients with cerebral amyloid angiopathy: a postmortem MRI study. Acta Neuropathol. 119, 291-302. doi: 10.1007/s00401-009-0615-z

Shatil, A. S., Matsuda, K. M., and Figley, C. R. (2016). A method for whole brain ex vivo magnetic resonance imaging with minimal susceptibility artifacts. Front. Neurol. 7:00208. doi: 10.3389/fneur.2016.00208

Shatil, A. S., Uddin, M. N., Matsuda, K. M., and Figley, C. R. (2018). Quantitative ex vivo MRI changes due to progressive formalin fixation in whole human brain specimens: longitudinal characterization of diffusion, relaxometry, and myelin water fraction measurements at 3T. Front. Med. 5:31. doi: 10.3389/fmed.2018.00031

Shepherd, T. M., Thelwall, P. E., Stanisz, G. J., and Blackband, S. J. (2009). Aldehyde fixative solutions alter the water relaxation and diffusion properties of nervous tissue. Magn. Reson. Med. 62, 26-34. doi: 10.1002/mrm.21977

Spatz, H. (1922). Über den eisennachweis im gehirn, besonders in zentren des extrapyramidal-motorischen systems. I. Teil. Z. Gesamte. Neurol. Psychiatr. 77, 261-390. doi: 10.1007/BF02865844 
Stüber, C., Morawski, M., Schäfer, A., Labadie, C., Wähnert, M., Leuze, C., et al. (2014). Myelin and iron concentration in the human brain: a quantitative study of MRI contrast. Neuroimage 93(Pt 1), 95-106. doi: 10.1016/j.neuroimage.2014.02.026

Stüber, C., Pitt, D., and Wang, Y. (2016). Iron in multiple sclerosis and its noninvasive imaging with quantitative susceptibility mapping. Int. J. Mol. Sci. 17:100. doi: 10.3390/ijms17010100

Sulzer, D., Mosharov, E., Talloczy, Z., Zucca, F. A., Simon, J. D., and Zecca, L. (2008). Neuronal pigmented autophagic vacuoles: lipofuscin, neuromelanin, and ceroid as macroautophagic responses during aging and disease. J. Neurochem. 106, 24-36. doi: 10.1111/j.1471-4159.2008.05385.x

Sun, H., Walsh, A. J., Lebel, R. M., Blevins, G., Catz, I., Lu, J.-Q., et al. (2015). Validation of quantitative susceptibility mapping with Perls' iron staining for subcortical gray matter. Neuroimage 105, 486-492. doi: 10.1016/j.neuroimage.2014.11.010

Tan, H., Liu, T., Wu, Y., Thacker, J., Shenkar, R., Mikati, A. G., et al. (2014). Evaluation of iron content in human cerebral cavernous malformation using quantitative susceptibility mapping. Invest. Radiol. 49, 498-504. doi: 10.1097/RLI.0000000000000043

Tashiro, K., Shiotani, S., Kobayashi, T., Kaga, K., Saito, H., Someya, S., et al. (2015). Cerebral relaxation times from postmortem MR imaging of adults. MRMS 14, 51-56. doi: 10.2463/mrms.2013-0126

Tatsumi, S., Shinohara, M., and Yamamoto, T. (2008). Direct comparison of histology of microbleeds with postmortem MR images: a case report. Cerebrovasc. Dis. 26, 142-146. doi: 10.1159/000139661

Terman, A., and Brunk, U. T. (2004). Lipofuscin. Int. Biochem. Cell Biol. 36, 1400-1404. doi: 10.1016/j.biocel.2003.08.009

Trujillo, P., Summers, P. E., Ferrari, E., Zucca, F. A., Sturini, M., Mainardi, L. T., et al. (2016). Contrast mechanisms associated with neuromelanin-MRI. Magn. Reson. Med. 78, 1790-1800. doi: 10.1002/mrm.26584

van Duijn, S., Nabuurs, R. J., van Duinen, S. G., and Natté, R. (2013). Comparison of histological techniques to visualize iron in paraffin-embedded brain tissue of patients with Alzheimer's disease. J. Histochem. Cytochem. 61, 785-792. doi: $10.1369 / 0022155413501325$

van Duijn, S., Nabuurs, R. J., van Rooden, S., Maat-Schieman, M. L., van Duinen, S. G., van Buchem, M. A., et al. (2011). MRI artifacts in human brain tissue after prolonged formalin storage. Magn. Reson. Med. 65, 1750-1758. doi: $10.1002 / \mathrm{mrm} .22758$

van Rooden, S., Maat-Schieman, M. L., Nabuurs, R. J., van der Weerd, L., van Duijn, S., van Duinen, S. G., et al. (2009). Cerebral amyloidosis: postmortem detection with human 7.0-T MR imaging system. Radiology 253, 788-796. doi: 10.1148/radiol.2533090490

van Veluw, S. J., Charidimou, A., van der Kouwe, A. J., Lauer, A., Reijmer, Y. D., Costantino, I., et al. (2016). Microbleed and microinfarct detection in amyloid angiopathy: a high-resolution MRI-histopathology study. Brain 139, 3151-3162. doi: 10.1093/brain/aww229

Vymazal, J., Brooks, R. A., Baumgarner, C., Tran, V., Katz, D., Bulte, J. W., et al. (1996). The relation between brain iron and NMR relaxation times: an in vitro study. Magn. Reson. Med. 35, 56-61. doi: 10.1002/mrm.1910350108

Wallace, M. N., Cronin, M. J., Bowtell, R. W., Scott, I. S., Palmer, A. R., and Gowland, P. A. (2016). Histological basis of laminar MRI patterns in high resolution images of fixed human auditory cortex. Front. Neurosci. 10:455. doi: 10.3389/fnins.2016.00455

Walsh, A. J., Lebel, R. M., Eissa, A., Blevins, G., Catz, I., Lu, J.-Q., et al. (2013). Multiple sclerosis: validation of MR imaging for quantification and detection of iron. Radiology 267, 531-542. doi: 10.1148/radiol.12120863

Wang, Y., and Liu, T. (2015). Quantitative susceptibility mapping (QSM): decoding MRI data for a tissue magnetic biomarker. Magn. Reson. Med. 73, 82-101. doi: $10.1002 / \mathrm{mrm} .25358$
Ward, R. J., Zucca, F. A., Duyn, J. H., Crichton, R. R., and Zecca, L. (2014). The role of iron in brain ageing and neurodegenerative disorders. Lancet Neurol. 13, 1045-1060. doi: 10.1016/S1474-4422(14)70117-6

Wiggermann, V., Hametner, S., Hernández-Torres, E., Kames, C., Endmayr, V., Kasprian, G., et al. (2017). Susceptibility-sensitive MRI of multiple sclerosis lesions and the impact of normal-appearing white matter changes. NMR Biomed. 30:e3727. doi: 10.1002/nbm.3727

Wisnieff, C., Ramanan, S., Olesik, J., Gauthier, S., Wang, Y., and Pitt, D. (2015). Quantitative susceptibility mapping (QSM) of white matter multiple sclerosis lesions: interpreting positive susceptibility and the presence of iron. Magn. Reson. Med. 74, 564-570. doi: 10.1002/mrm.25420

Yao, B., Li, T. -Q., Gelderen P. v., Shmueli, K., de Zwart, J. A., and Duyn, J. H. (2009). Susceptibility contrast in high field MRI of human brain as a function of tissue iron content. Neuroimage 44, 1259-1266. doi: 10.1016/j.neuroimage.2008.10.029

Ye, F. Q., Martin, W. R., and Allen, P. S. (1996). Estimation of the iron concentration in excised gray matter by means of proton relaxation measurements. Magn. Reson. Med. 35, 285-289. doi: 10.1002/mrm.1910 350304

Yelnik, J., Bardinet, E., Dormont, D., Malandain, G., Ourselin, S., Tand,é, D., et al. (2007). A three-dimensional, histological and deformable atlas of the human basal ganglia. I. Atlas construction based on immunohistochemical and MRI data. Neuroimage 34, 618-638. doi: 10.1016/j.neuroimage.2006.09.026

Yong-Hing, C. J., Obenaus, A., Stryker, R., Tong, K., and Sarty, G. E. (2005). Magnetic resonance imaging and mathematical modeling of progressive formalin fixation of the human brain. Magn. Reson. Med. 54, 324-332. doi: $10.1002 / \mathrm{mrm} .20578$

Zaleski, S. S. (1887). Das Eisen der Organe bei Morbus maculosus Werlhofii. Arch. Exp. Pathol. Pharmakol. 23, 77-90. doi: 10.1007/bf01837929

Zecca, L., Bellei, C., Costi, P., Albertini, A., Monzani, E., Casella, L., et al. (2008). New melanic pigments in the human brain that accumulate in aging and block environmental toxic metals. Proc. Natl. Acad. Sci. U.S.A. 105, 17567-17572. doi: 10.1073/pnas.0808768105

Zecca, L., Youdim, M. B., Riederer, P., Connor, J. R., and Crichton, R. R. (2004). Iron, brain ageing and neurodegenerative disorders. Nat. Rev. Neurosci. 5, 863-873. doi: 10.1038/nrn1537

Zheng, W., Haacke, E. M., Webb, S. M., and Nichol, H. (2012). Imaging of stroke: a comparison between X-ray fluorescence and magnetic resonance imaging methods. Magn. Reson. Imag. 30, 1416-1423. doi: 10.1016/j.mri.2012.04.011

Zheng, W., Nichol, H., Liu, S., Cheng, Y. C., and Haacke, E. M. (2013). Measuring iron in the brain using quantitative susceptibility mapping and X-ray fluorescence imaging. Neuroimage 78, 68-74. doi: 10.1016/j.neuroimage.2013.04.022

Zucca, F. A., Segura-Aguilar, J., Ferrari, E., Muñoz, P., Paris, I., Sulzer, D., et al. (2017). Interactions of iron, dopamine and neuromelanin pathways in brain aging and Parkinson's disease. Prog. Neurobiol. 155, 96-119. doi: 10.1016/j.pneurobio.2015.09.012

Conflict of Interest Statement: The authors declare that the research was conducted in the absence of any commercial or financial relationships that could be construed as a potential conflict of interest.

Copyright (c) 2019 De Barros, Arribarat, Combis, Chaynes and Péran. This is an open-access article distributed under the terms of the Creative Commons Attribution License (CC BY). The use, distribution or reproduction in other forums is permitted, provided the original author(s) and the copyright owner(s) are credited and that the original publication in this journal is cited, in accordance with accepted academic practice. No use, distribution or reproduction is permitted which does not comply with these terms. 